\title{
Quality models for web services: A systematic mapping
}

\author{
Marc Oriol*, Jordi Marco, Xavier Franch \\ Universitat Politècnica de Catalunya - BarcelonaTech, ESSI - UPC. 08034 Barcelona, c/Jordi \\ Girona 1-3, Spain \\ \{moriol, jmarco\}@lsi.upc.edu, franch@essi.upc.edu
}

* Corresponding author. Address: Universitat Politècnica de Catalunya - BarcelonaTech, ESSI UPC. 08034 Barcelona, c/Jordi Girona 1-3, Omega Building S-206, Spain.

e-mail: moriol@Isi.upc.edu Telf: +34 934137862.

\begin{abstract}
Context: Quality of Service (QoS) is a major issue in various web service related activities. Quality models have been proposed as the engineering artefact to provide a common framework of understanding for QoS, by defining the quality factors that apply to web service usage.

Objective: The goal of this study is to evaluate the current state of the art of the proposed quality models for web services, specifically: (1) which are these proposals and how are they related; (2) what are their structural characteristics; (3) what quality factors are the most and least addressed; and (4) what are their most consolidated definitions.

Method: We have conducted a systematic mapping by defining a robust protocol that combines automatic and manual searches from different sources. We used a rigorous method to elicitate the keywords from the research questions and a selection criteria to retrieve the final papers to evaluate. We have adopted the ISO/IEC 25010 standard to articulate our analysis.

Results: We have evaluated 47 different quality models from 65 papers that fulfilled the selection criteria. By analyzing in depth these quality models, we have: 1) distributed the proposals along the time dimension and identified their relationships; 2 ) analysed their size (visualizing the number of nodes and levels) and definition coverage (as indicator of quality of the proposals); 3) quantified the coverage of the different ISO/IEC 25010 quality factors by the proposals; 4 ) identified the quality factors that appeared in at least $30 \%$ of the surveyed proposals and provided the most consolidated definitions for them.

Conclusions: We believe that this panoramic view on the anatomy of the quality models for web services may be a good reference for prospective researchers and practitioners in the field and especially may help avoiding the definition of new proposals that do not align with current research.
\end{abstract}

\section{Keywords}

Quality Model, Quality of Service, Web Service, Systematic Mapping

\section{Introduction}

Web services are software components used through the internet that can be integrated into more complex distributed applications[1]. Usually, web services are third-party software made available by service providers. Therefore, these distributed applications based on web services, commonly known as service-based systems, rely on these providers to ensure that their services comply with the agreed Quality of Service (QoS). The analysis of QoS-related issues becomes crucial for several web service activities, from service selection to service decommission and service-based systems adaptation; therefore, QoS is currently a leading research topic in the field [2]. 
In this regard, all kind of proposals and approaches for different activities involving the definition and analysis of QoS in web services have emerged. Maybe the first question that may be raised is: what are the facets that compose QoS? In other words, what criteria can make a "good" service? The answer to this question requires determining a shared universe of discourse, a common framework of understanding upon which different actors may communicate effectively, without ambiguities. Quality models are the engineering artifacts that have been proposed to structure and standardize the concepts and definitions of the quality factors of the QoS in web services. However, as it happens in many other software engineering areas, there does not exist a single quality model agreed by the community, instead many adhoc proposals have emerged in the last decade. These proposals may diverge in several matters: addressed facets, size, structure, terminology, underlying principles, etc. At this point of time, it may be argued that it is necessary an effort to assess the current state of the art of quality models for web services. It is important to identify and relate the existing proposals, assess them with respect to some criteria and conclude which is the most consolidated body of knowledge and on the contrary, which are the most remarkable gaps to bridge.

The aim of this study is to identify and evaluate the different quality models for web services proposed in the literature. We have conducted a systematic mapping to accurately retrieve and analyse the different quality models by defining and conducting a rigorous protocol following the guidelines described in [3]. As a result of such research, we have been able to evaluate the current state of the art in quality models and identify the strengths and weaknesses of its current status.

The remainder of the paper is structured as follows. An introductory background in quality models and systematic mapping is presented in Section 2. In Section 3, the systematic mapping protocol is described. In Section 4, we present the results of the systematic mapping. Threats to validity to our study are analyzed in Section 5. Finally, in Section 6, we present the conclusions and future work.

\section{Background}

The background is composed of two subsections. The first one is the background on the domain, in which we briefly introduce the concepts of quality model and quality of service. The second subsection provides the basic notions of the instrument chosen for conducting our study, namely a systematic mapping.

\subsection{Quality Models}

As the ISO/IEC 25010 software quality standard states, quality models are useful for specifying requirements, establishing measures and performing quality evaluations [4]. A quality model is a hierarchically decomposed set of quality attributes of software. In IEEE-610 [5], a quality attribute is defined as "a feature or characteristic that affects an item's quality."

There exist many proposals of general-purpose quality models for software systems. They differ on the terminology that they use, the set of quality attributes they define, and the structure of the quality model. The most widely adopted one is the ISO/IEC series of quality standards, especially the 9126 and its replacement, 25010 [4]. Both of them include a concrete proposal of quality model that classifies software quality into a structured set of high-level characteristics and sub-characteristics. Fig. 1 shows the 25010 proposal. 


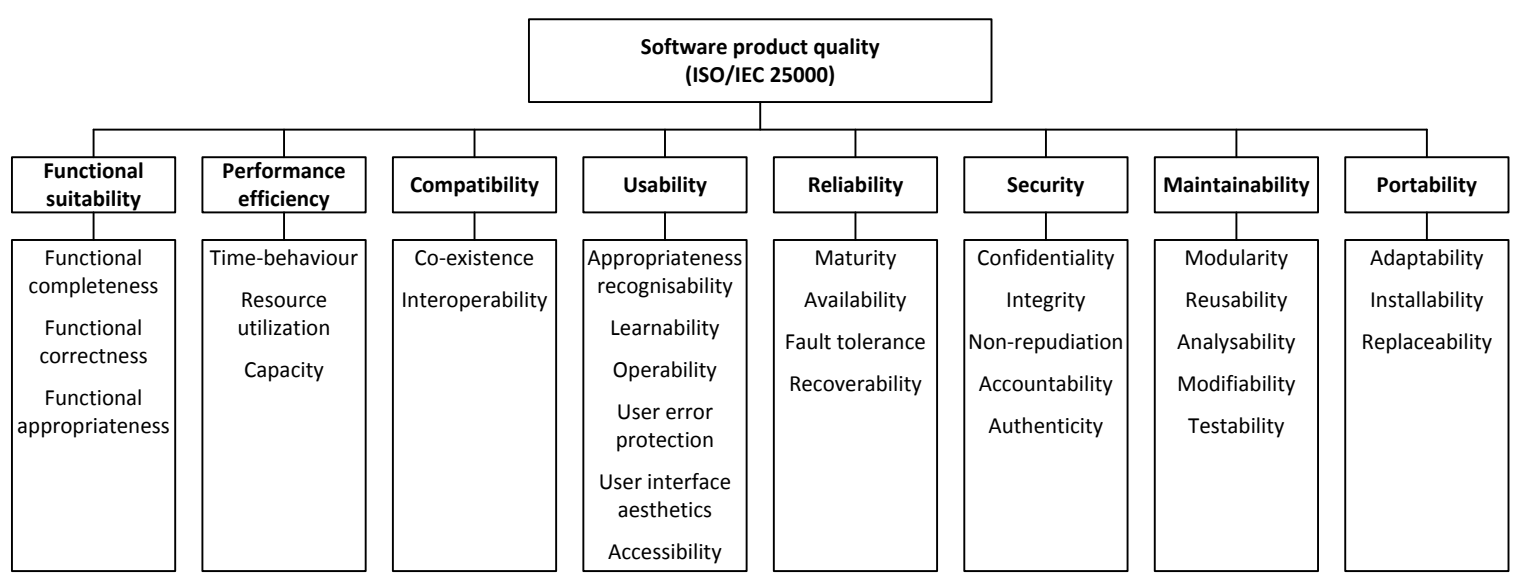

Figure 1: ISO/IEC 25010 proposal of quality model for software products

When it comes to quality models for web services, the notion of Quality of Service (QoS) emerges. As stated in ISO 8402 [6], the QoS is "the totality of features and characteristics of a product or service that bear on its ability to satisfy stated or implied needs". Generic standards as ISO/IEC 25010 might not accommodate completely into the web service domain. For instance, Installability is usually not applicable to web services because they are executed remotely at the server side. This is the reason why other quality models that are specific for web services have been proposed, as will be shown in this paper.

On the other hand, the high-level quality characteristics defined in generic quality models like the ISO/IEC ones do not provide quantitative or qualitative measurements. For instance, it cannot be stated that the time behaviour of a web service is $140 \mathrm{~ms}$. Therefore, it is necessary to decompose them into fine-grained concepts as response time, latency or execution time, among others. When these concepts are clearly defined in measurable terms, they are usually known as quality metrics. As defined by Burnstein, "a quality metric is a quantitative measurement of the degree to which an item possesses a given quality attribute" [7]. An example of quality metric is the average response time during a time interval. Fig. 2 makes explicit the relationship between the different concepts defined so far. Following the hierarchy of a quality model, QoS is depicted on top, which is decomposed in quality characteristics, quality attributes and metrics progressively.

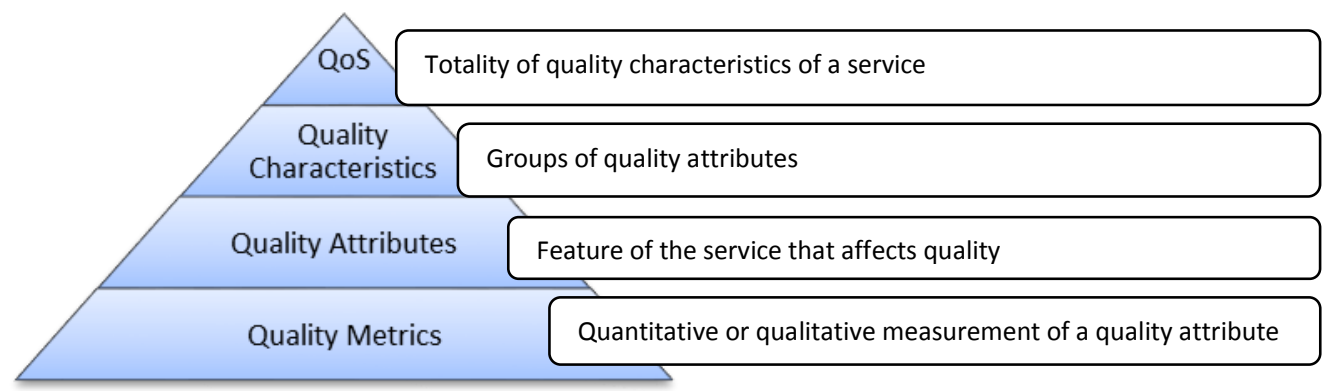

Figure 2: Hierarchy of quality concepts

Despite the clear theoretical difference between these concepts, the line differentiating each of them can be sometimes blurring. Consequently, some contributions use them arbitrarily. Some other works consider quality attributes and quality characteristics as synonyms, and hence, they use the terms quality groups [8] or QoS categories [9] in order to group the quality attributes. In this paper, we also introduce the term "quality factor" as a generalization of the terms "quality characteristic" and "quality attribute", because sometimes we need to refer to both of them together. 


\subsection{Systematic Mappings}

To conduct any type of literature study in an accurate and objective manner, it is necessary to use a precise and rigorous methodology. For such a purpose, this work uses the guidelines proposed by B. Kitchenham [3]. These guidelines have been derived from other existing studies used by medical researchers and adapted to reflect the specific problems of software engineering research. Since their inception, these guidelines have been widely used by software engineering researchers and when applied properly, they drastically reduce the risk of bias and incompleteness in the review results.

The stages of the methodology, as defined by B. Kitchenham [3], are as follows:

- Planning the review: activities performed before conducting the review. These activities include the identification of the need for a review, the statement of the research questions that the review is intended to answer, and the definition of the protocol that specifies the criteria that will be used to perform the review (e.g. search keywords, bibliographic databases, selection criteria, etc.).

- Conducting the review: activities that constitute the execution of the review and follow the protocol as defined in the previous phase. These activities include the identification of research, the selection of primary studies, the study of quality assessment, data extraction and synthesis.

- Reporting the review: activities to report the results of the review. It includes the specification of a dissemination mechanism, the format of the report, and its evaluation.

This methodology may be applied to the two main existing types of systematic literature studies, namely systematic literature reviews (SLR) [10] and systematic mappings [11]. Whilst SLRs investigate in detail some insightful research question, a systematic mapping is a method to review, classify, and structure papers related to a specific topic. The goal of a systematic mapping is to obtain an overview of existing approaches, outlining the coverage of the research field and its gaps. Given the objective of our study, it can be classified as a systematic mapping.

The next two sections report the two first stages applied to our study.

\section{Planning the Review}

\subsection{Identification of the need for a review}

As Kitchenham states, prior to undertaking any systematic literature study, we ensured that such a study is necessary by searching for others in the subject and assessing their quality through a quality assessment checklist.

There is no procedure defined in [3] in order to implement this search. But to make this step also systematic, we applied two tactics. First, to increase the number of results, we searched not only other systematic literature studies, but all type of reviews and state of the art documents, regardless of the methodology followed for developing them. Second, we followed a procedure analogous to the main search of our systematic mapping. That is, we defined a search protocol to identify other reviews. Such protocol is based on the protocol defined in the main search, which will be explained in the following sections. Hence, we searched for other reviews once the systematic mapping protocol was defined and before the systematic mapping was conducted. In a glance, we combined automatic and manual searches to the same databases and resources, using the same keywords with the addition of the following terms: "state of the art", "SLR", 
"survey", "review" and "systematic mapping". As a result, in the automatic search we found 47 papers fulfilling the search criteria (details are presented in [12]). However, after inspecting them, we found that none of them presented a review on quality models for web services. Similarly, with respect to the manual search, we found 1 paper fulfilling the search criteria, which was also discarded for the same reason.

Therefore, after performing the searches, we did not find any literature study on quality models for web services.

\subsection{The research questions}

The next step of the review is the formulation of the research question which, in turn, will drive the review methodology. To formulate it, we followed the PICO structure [3]. PICO is an acronym which stands for Population, Intervention, Comparison and Outcome. It consists of explicitly identifying these concepts in the construction of the research question in order to derive later the keywords to perform the search. In our case, though, the Comparison is more a kind of general analysis of the field, since we do not aim at ranking the proposals found or to compare to some other existing approach. The research question that we aim at is:

In the field of web services (P), do software quality models (I) proposed so far provide an adequate and structured set of quality factors to define their quality of service (0)?

Since this main research question is very general, we refined it into finer-grained subquestions. First, we want to identify the proposals in the field, find their interrelationships and distribute them along time to find any significant trend (RQ1.1). Second, we want to make explicit the main characteristics of these quality models, in terms of size, structure and completeness (RQ1.2). Third, we want to find which is the scope of these quality models. Namely, what are the quality factors that attract more attention from researchers and also which attract less, since both things together may help to understand priorities of researchers and eventually some research gaps (RQ1.3). Last, we want to uncover the agreed body of knowledge among the different proposals. Particularly, we aim at identifying the most recurrent definitions of quality factors in these proposals, which in some sense could be considered as the starting point of any new future proposal (RQ1.4). The resulting subquestions can be stated then as:

RQ 1.1 What is the chronological overview of the research done so far in quality models for web services?

RQ 1.2 What are the characteristics of the proposed quality models?

RQ 1.3 Which quality factors are the most addressed in these quality models? Which are the least addressed?

RQ 1.4 What are the most consolidated quality factors?

\subsection{The Protocol}

\subsubsection{Bibliographic sources}

In any systematic mapping, the search process can be either automatic through the usage of bibliographic databases or manual through gathering the works from specific known journals and conferences of the target field. Kitchenham et al. analysed in [13] the advantages and

\footnotetext{
${ }^{1}$ We use this term, more general than "systematic literature review", to make the search more robust with respect to terminological variations.
} 
drawbacks of both approaches through a case study. From their analysis, we decided to combine both of them by performing an automatic search to some selected databases and additional manual searches to the most relevant conferences and journals if they were missing. The selected databases, were ISI Web of Science (WoS), IEEE Xplore and ACM Digital Library.

To assess the quality and not only the quantity of the research works, we verified that the following list of journals and conferences relevant in the field of web services were present:

- Journals: ACM - Computing Surveys, ACM - TOIT, ACM - TOSEM, ACM - TWEB, Elsevier Advances in Engineering Software, Elsevier - IST, Elsevier - JSS, Elsevier - JWS, IEEE Computer, IEEE - Internet Computing, IEEE - Software, IEEE - TSE, IGI global JWSR, Springer - SQJ and Springer - WWW.

- Conferences: ASE, CAiSE, E-Science, FSE, GRID, ICSE, ICSOC, ICWS, ISSTA, SCC, WISE, WWW.

The list of journals was obtained from the top ranked journals in services, software engineering and information systems engineering based on their JCR impact factor. Similarly the list of conferences was obtained from the top ranked conferences based on the CORE index (http://core.edu.au/index.php/categories/conference\%20rankings), selecting those which had a CORE-A status ${ }^{2}$.

Through checking the list of editions of each conference and journal from 2001 to 2012, we verified that the journals were indexed with all their editions published in ISI WoS. However, with respect to conferences, we identified that, although all these conferences were indexed (with the exception of FSE), most of them didn't have all the editions present in the database. Hence, in the next stage of the systematic mapping, we had to perform a manual search in these missing editions (see Table 1).

Table 1: Coverage of manual and automatic searches.

\begin{tabular}{|c|c|c|c|c|c|c|c|c|c|c|c|c|}
\hline & & & & & & & & \multicolumn{5}{|c|}{ Coverage of Manual and Automatic searches } \\
\hline & 2001 & 2002 & 2003 & 2004 & 2005 & 2006 & 2007 & 2008 & 2009 & 2010 & 2011 & 2012 \\
\hline ASE & Auto. & Auto. & Auto. & Auto. & Auto. & Auto. & Auto. & Auto. & Auto. & Manual & Manual & Manual \\
\hline CAISE & Auto. & Auto. & Manual & Manual & Manual & Manual & Manual & Manual & Manual & Manual & Auto. & Manual \\
\hline E-Science & - & - & - & - & Auto. & Manual & Auto. & Manual & Manual & Manual & Manual & Manual \\
\hline FSE & Manual & Manual & Manual & Manual & Manual & Manual & Manual & Manual & Manual & Manual & Manual & Manual \\
\hline GRID & Manual & Manual & Manual & Manual & Manual & Auto. & Auto. & Auto. & Manual & Manual & Manual & Auto. \\
\hline ICSE & Auto. & Auto. & Auto. & Auto. & Auto. & Auto. & Auto. & Manual & Auto. & Auto. & Auto. & Auto. \\
\hline ICSOC & - & - & Manual & Manual & Manual & Manual & Auto. & Manual & Manual & Manual & Auto. & Manual \\
\hline ICWS & - & - & Manual & Auto. & Manual & Auto. & Auto. & Auto. & Auto. & Manual & Manual & Manual \\
\hline ISSTA & - & Manual & - & Manual & - & Manual & Manual & Auto. & Auto. & Manual & Manual & Manual \\
\hline SCC & - & - & - & Auto. & Auto. & Manual & Auto. & Auto. & Auto. & Manual & Manual & Manual \\
\hline WISE & Auto. & Auto. & Auto. & Auto. & Auto. & Auto. & Auto. & Auto. & Auto. & Auto. & Auto. & Manual \\
\hline www & Auto. & Auto. & Auto. & Manual & Auto. & Auto. & Auto. & Auto. & Manual & Manual & Manual & Manual \\
\hline
\end{tabular}

The guidelines recommend also retrieving not only research papers from conferences and journals, but also deliverables and other documents from the grey literature. To do so, we also performed manual searches in the deliverables, standards and working drafts from the following international consortiums and networks which are well established in the community:

- DMTF: Distributed Management Task Force (http://www.dmtf.org/)

- ETSI: European Telecommunications Standards Institute (http://www.etsi.org/)

- IETF: Internet Engineering Task Force (http://www.ietf.org/)

\footnotetext{
${ }^{2}$ There is no standard procedure to select the list of conferences. Although the CORE-A index, as any other, can raise controversy, we consider that it is a good indicator for our purposes.
} 
- NESSI: Networked European Software and Services Initiative (http://www.nessieurope.com)

- OASIS: Organization for the Advancement of Structured Information Standards (https://www.oasis-open.org)

- OGF: Open Grid Forum (http://www.gridforum.org/)

- OpenGroup (http://www.opengroup.org/)

- W3C: World Wide Web Consortium. (http://www.w3.org/)

- WS-I: Web Services Interoperability Organization (http://www.ws-i.org/)

\subsubsection{Keywords used}

The keywords used for the search were retrieved from the PICO terms of the research questions. Particularly we extracted them from the Population and Intervention. In the guidelines [3], it is suggested to extract the keywords also from the Comparison and Outcome, which is the common procedure in the field of medicine. However, as it stated also by Kitchenham in [14] and identified in other SLRs [15] and systematic mappings [11], this is not always applicable. For instance, it is applicable in SLRs when performing a comparison between two already known different approaches [3]. However, in our case we evaluated the different proposed approaches in the literature, which would imply that we should know beforehand the proposed approaches to perform the searches. Similarly, the outcome in our research questions is not based on a particular measurement and hence it cannot be included as a keyword.

From each term of the Population and Intervention of the research questions, we identified a set of variants and acronyms:

\begin{tabular}{|l|l|}
\hline P: Web service & P1 Terms: “web service", webservice, "web services", webservices, WS \\
\hline I: Quality models & $\begin{array}{l}\text { I1 Terms: "quality model”, "quality models", QM, "quality ontology", "quality } \\
\text { ontologies", QO, "quality of service", "quality of services", QoS }\end{array}$ \\
\hline
\end{tabular}

To build the query string, the terms inside $\mathbf{P}$ and $\mathbf{I}$ are composed through an OR connector (e.g. P: "web service" OR "webservice" OR "web services" OR "webservices" OR WS). Then P and I are composed through an AND connector. The resulting query string is then:

("web service" OR webservice OR "web services" OR webservices OR WS) AND

("quality model" OR "quality models" OR QM OR "quality ontology" OR "quality ontologies" OR QO OR "quality of service" OR "quality of services" OR QoS)

which can be simplified into the following version:

(webservice? OR "web service?" OR "WS") AND

("quality model?" OR QM OR "quality ontology" OR "quality ontologies" OR QO OR "quality of service?" OR QoS)

In particular, we simplified singular and plural forms by using the '?' wildcard due to known limitations of the length of the query in ISI Web of Science. Although the resulting terms are not fully equivalent, we considered the probability of the wildcard delivering results other than plurals to be very low. The simplified query string was applied to search into ISI Web of Science. To search into IEEE Xplore and ACM DL, the original query string was used.

\subsubsection{Selection criteria}


The search was conducted by title, abstract and keywords in ISI Web of Science, IEEE Xplore and ACM DL. For the conference editions not in the aforementioned databases (see Table 1), we conducted manually the same search over title, abstract and keywords.

After retrieving the results, we followed several steps applying selection criteria to filter the candidates:

1. Selection by title: The objective of this first filter is to quickly identify and remove noise from the results. After this selection, documents whose scope is clearly unrelated to quality models for web services were removed.

2. Selection by abstract: At this stage, we discarded all those works that although being related to quality models, didn't present a quality model as contribution of the paper.

3. Selection by full paper (fast reading): At this point, we removed the papers which didn't accomplish properly the following inclusion criteria: (1) presenting a quality model as one of the contributions of the paper; (2) defining explicitly the quality model.

4. Literature from organizations: Deliverables or similar documents from the international consortiums and organizations identified above cannot be conducted through the same process as they might lack of an abstract or mechanisms to perform a systematic search. Hence the search of these documents against the list of resources provided in the web pages of the organizations was conducted manually. The works satisfying the same inclusion criteria as Step 3 were added into the systematic mapping.

5. Addition of further work (snowballing): During the systematic mapping process, other works were included through the process of snowballing. In this sense, we included those works that were the basis for the development of the quality model proposed in the retrieved papers. The works satisfying the same inclusion criteria as Step 3 were added into the systematic mapping.

From our searches we retrieved 1004 papers automatically from ISI WoS, 1105 from IEEE Xplore and 189 from ACM DL. From the total of 2298 papers, 438 were removed as they were repeated in the selected databases, leading to 1860 papers found automatically. Then, we added 28 papers found from the manual searches from the selected journals and conferences. From the initial set of 1888 papers retrieved, 518 were discarded by title and 1031 papers were excluded by abstract; resulting in 339 papers to evaluate by full paper. From them, 15 papers were initially not available, and after contacting the authors, we were able to retrieve only 5 . Hence, 10 papers were not accessible. Then we evaluated the works by full paper, 284 papers were discarded, resulting in 45 papers to include in the systematic mapping.

Then 5 documents found in the literature from the listed organizations were added. Finally, 15 additional papers were added through snowballing resulting in the final 65 papers. The complete list of papers involved in the search process is available at [12]. A summary of the selection process is depicted in Fig. 3.

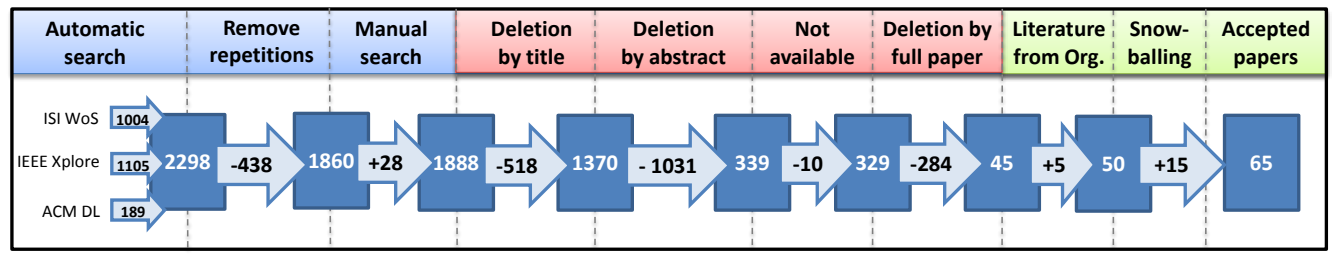

Figure 3: Selection process

\section{Results of the review}


We conducted the review according to the criteria and protocols defined in the previous section. First, we read the 65 selected works individually and consolidated the information therein. The 65 works present 47 different proposals of quality models (i.e. some proposals are explained in more than one work) that we summarized in tables that are included in [12]. Each table includes the name of the quality factor, an identifier system that reproduces the hierarchy and the definition as given by the authors. Next we address our research questions on the basis of this information.

\subsection{What is the chronological overview of the research done so far in quality models for web services?}

Quality models for web services have been an increasingly addressed research topic from 2001 to the current days. Fig. 4 shows the number of papers considered in two-year periods starting at 2001. As shown there, the number of papers found (i.e. fulfilling the search criteria) has been increasing as well as the number of papers that have been selected in our systematic mapping (i.e. fulfilling the topic and quality criteria). However, the period 2011-2012 shows a decreased amount of papers on both aspects with respect to the previous period.

On the other hand, as shown in Fig. 4, the number of papers found has been increasing at a faster pace than the selected ones. This fact implies that, although the topic has been gaining increasing attention, the actual proposals of new or improved quality models have not been increasing to the same degree.

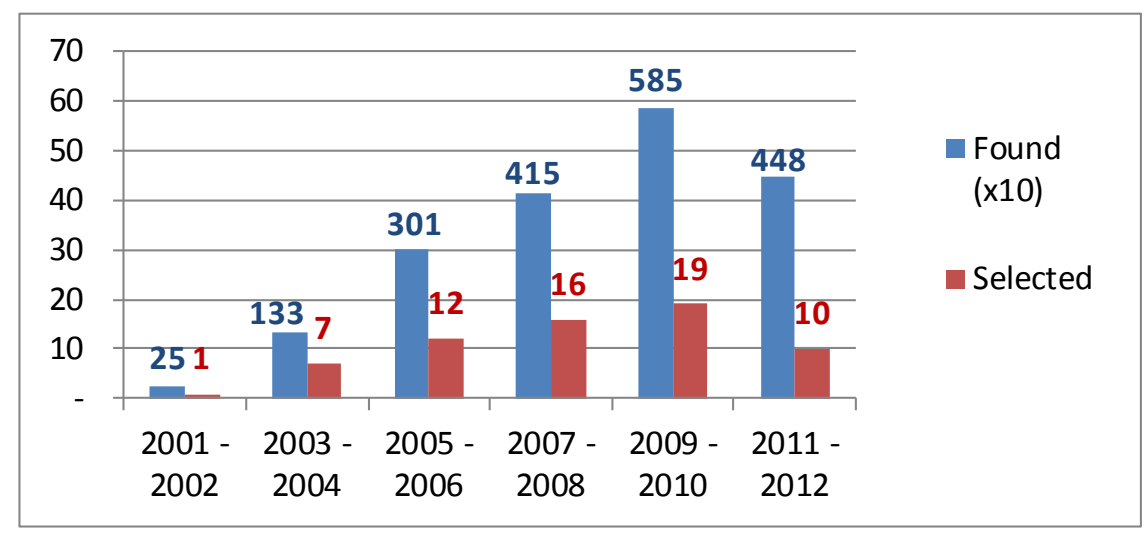

Figure 4: List of papers found and selected by 2-year period.

Considering the 47 proposals and not the 65 individual papers, the first quality model we found specifically designed for web services was issued in 2002 [16]. Since then, we identified that many proposals have been developed based on other previous quality models, sharing many concepts and definitions. Most of the oldest quality models have been updated or enhanced by other researchers. In order to picture the evolution of such proposals, and depict the research done in quality models, we have built the genealogical tree, which is shown in Fig. 6.

Some relevant observations follow:

- Contrary to what could be expected, most of the proposals do not take into consideration the standards ISO 9126-1 [17] or ISO/IEC 25010 [4] for the development of the quality model, with the exceptions of the S-Cube Quality Reference Model [8], WSQM [18], [19], Abramowicz et al. [20], Yin et al. [21], GESSI [22]-[24] and Nadanam et al. [25].

- $56 \%$ of the approaches have not considered other quality models for web services in their definition. Although this could be considered acceptable for the oldest ones, it is not so acceptable for the most recent ones. For instance, if we consider 2007 onwards, there are 
fewer proposals that build on top of others (13) than new proposals (20). We think that this is an indicator that a study like the systematic mapping undertook in this paper may help researchers in the field to be more aware of the current state of the art.

- Symmetrically, $26 \%$ of the approaches have influenced the definition of other quality models. If we focus on particular proposals, the oldest quality models have had the biggest impact. The most used quality model is from S. Ran [26] which has been used to develop 8 quality models, followed by E. Maximilien and P. Singh [27] (influencing 6 quality models), and both IBM [16] and W3C QoS [28] (influencing 5 quality models each). Still a few more quality models have influenced the definition of other proposals [18]-[21], [29]-[35] . Please note that these numbers do not consider transitivity, i.e. if a quality model $A$ influenced the definition of a quality model $B$ and $B$ influenced the definition of another quality model $C$, we have not counted $A$ in the influencing set of $C$.

- The approach which takes more quality models into consideration for the development of its proposal is BREIN QoS Ontology [36], [37] (with 6 proposals) followed by WSMO-QoS [38], M. Comuzzi and B. Pernici [39] and Yin et al. [21] (with 4 proposals each). To a lesser extent, also Yeom et al. [40], Tsesmetzis et al. [41], [42] , E. Maximilien and P. Singh [27], Guimarães [43], and Soomro and Song [44] (with 2 proposals each). It is worth to clarify that this analysis is scoped to the relationship between the quality models in the field of web services. Other models (e.g. UML profiles) have not been considered although they have been taken into account for the development of certain quality models (e.g. S-Cube Quality Reference Model [8]).

- The approach that reflects the longest evolution (i.e. more quality models in its path) is Yin et al. [21], whose evolution path goes through 7 quality models starting from 2003.

- Last, we address endorsement of the proposals. The first quality models were developed by organizations such as IBM [16] and W3C [28]. More recent quality models such as WSMO are developed by the WSMO working group and supported by W3C [45], [46]; the OASIS group has also developed a quality model named WSQM [18], [19]. On the other hand, some quality models have been developed by European Networks, as S-Cube [8], or European Projects as BREIN [36], [37]. Fig. 5 summarizes the overall results.

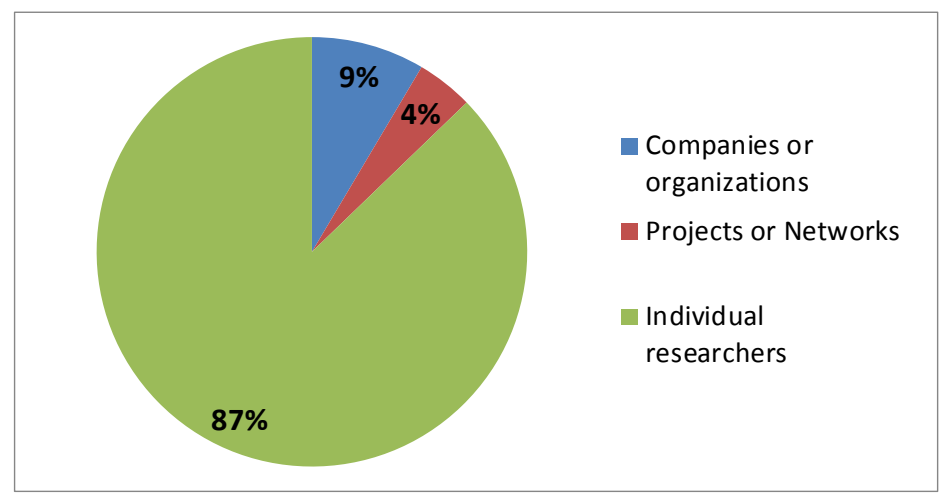

Figure 5: Endorsement of the proposed quality models 


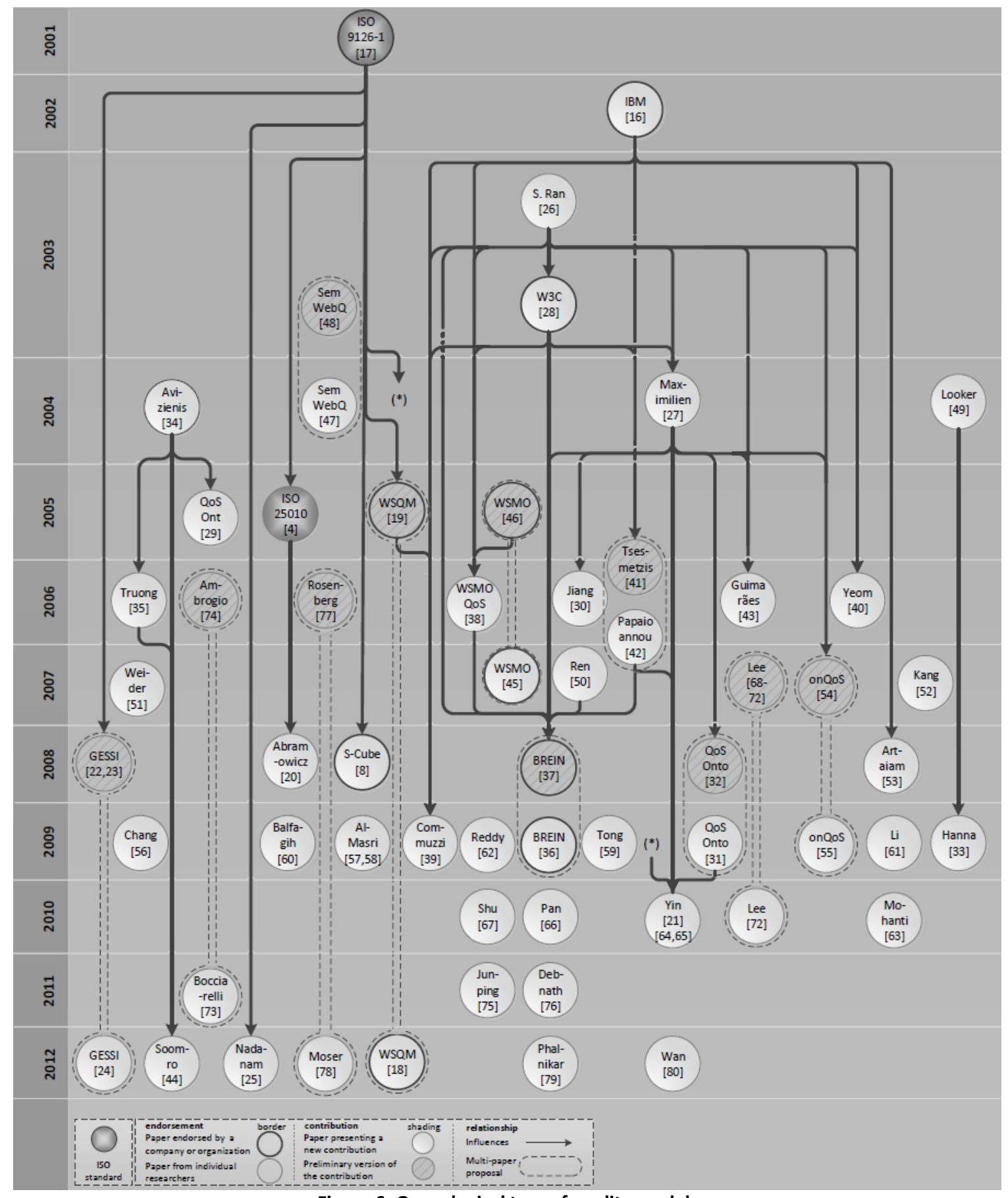

Figure 6: Genealogical tree of quality models

\subsection{What are the characteristics of the proposed quality models?}

To answer this question, we evaluate the structural characteristics of the proposed quality models in terms of its size (quantity) and definition coverage (quality).

- Size: We evaluated the size by the amount of nodes (representing quality factors) present in the quality models and its level of depth. Through this criteria, the most extensive quality models are the S-Cube Quality Reference Model [8] with 66 nodes, followed by GESSI [22][24] with 57 and Truong et al. [35] with 45 nodes. In all these cases, the presented quality models have a 3-level depth. In this regard, we found a clear correlation between the number of nodes and the depth level (See Fig. 7). For instance, quality models presenting 
only between 6 and 10 nodes, were usually developed in 1-level depth ( 6 proposals). In the mid-range area, quality models of between 26 and 30 nodes were usually developed in a 2level depth ( 9 proposals) and larger quality models (more than 30 nodes) were more often presented in a 3-level depth. On average, the proposed quality models have 24,38 nodes and a depth level of 2,23.

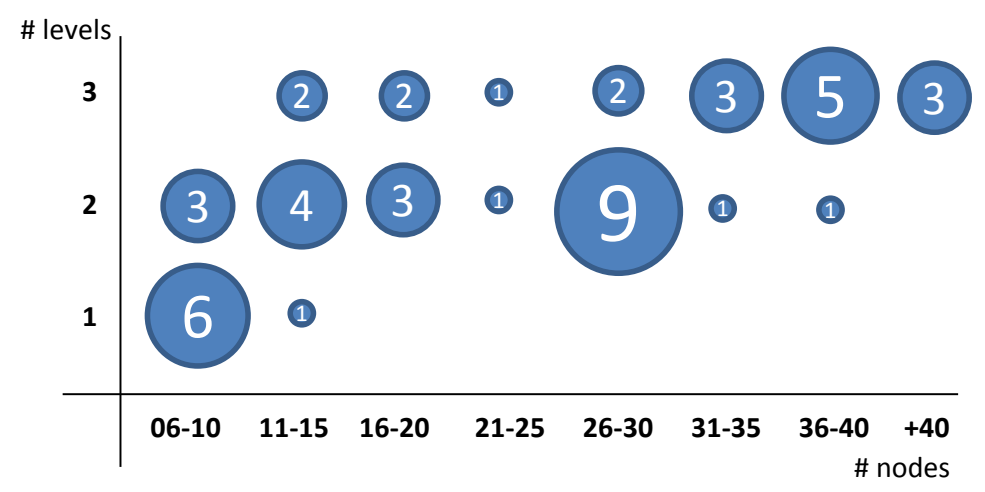

Figure 7: Correlation map between nodes and levels.

- Definition completeness: A narrow majority of the presented proposals, up to $51 \%$, have a unique and consistent definition for all the quality factors that are present in the quality model (i.e. 100\% definition completeness), either by explicitly defining the quality factor in the paper or by referencing to the paper which has the definitions (See Fig. 8).

The remaining proposals present problems of different scale: (1) some quality factors are not defined; (2) the quality model is based on several quality models and although they are referenced, it is not specified which is the chosen definition for each quality factor, leading to different definitions which are not consistent with each other; (3) the definitions on some quality factors are too vague or ambiguous.

Following these criteria, $15 \%$ of quality models have a definition completeness between $80 \%$ and $99 \%$ (i.e. between $80 \%$ and $99 \%$ of the quality factors are defined without any of the aforementioned problems), $15 \%$ of the quality models have a definition completeness between $60-79 \%$, and $19 \%$ of the proposals have a definition completeness below $40 \%$.

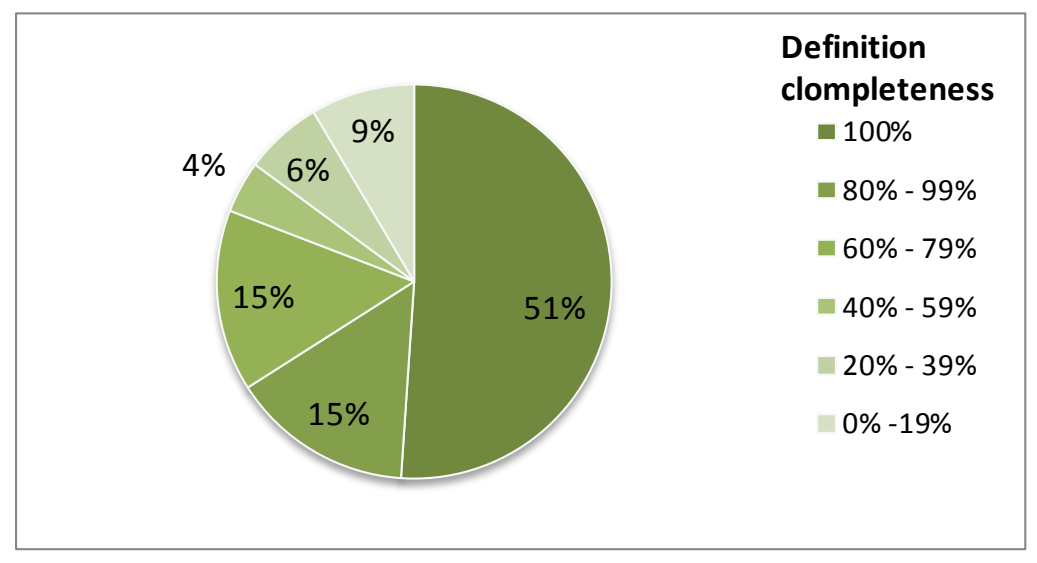

Figure 8: Percentage of Quality models with their definition completeness. 
Table 2 summarizes the most significant information about the 47 surveyed proposals in the answer of these two first research questions.

Table 2: Characteristics of the quality models.

\begin{tabular}{|c|c|c|c|c|c|}
\hline Proposal & Year & Develop. Method & Deph Level & Nodes & $\begin{array}{c}\text { Definition } \\
\text { completeness }\end{array}$ \\
\hline IBM [16] & 2002 & From scratch & 2 & 9 & $100 \%$ \\
\hline S. Ran [26] & 2003 & From scratch & 3 & 35 & $100 \%$ \\
\hline W3C Qos [28] & 2003 & Based on [26] & 2 & 30 & $100 \%$ \\
\hline E. Maximilien and P. Singh [27] & 2004 & Based on $[26,28]$ & 2 & 26 & $100 \%$ \\
\hline A. Avizienis et al. [34] & 2004 & From scratch & 3 & 15 & $100 \%$ \\
\hline SemWebQ [47], [48] & 2003-2004 & From scratch & 3 & 15 & $100 \%$ \\
\hline Looker et al. [49] & 2004 & From scratch & 2 & 7 & $100 \%$ \\
\hline QoSOnt [29] & 2005 & Based on [34] & 1 & 6 & $100 \%$ \\
\hline WSMO-QoS [38] & 2006 & Based on $[46,28,26,16]$ & 2 & 27 & $88 \%$ \\
\hline S. Jiang and F. Aagesen [30] & 2006 & Based on [27] & 2 & 16 & $81 \%$ \\
\hline G. Yeom el al. [40] & 2006 & Based on $[26,16]$ & 3 & 39 & $100 \%$ \\
\hline D.T. Tsesmetzis et al. [41], [42] & 2006 & Based on $[28,16]$ & 2 & 27 & $93 \%$ \\
\hline Guimarães and Beatriz [43] & 2006 & Based on [26][27] & 1 & 15 & $100 \%$ \\
\hline Truong et al. [35] & 2006 & Based on [34] & 3 & 45 & $71 \%$ \\
\hline Ren et al. [50] & 2007 & From scratch & 2 & 26 & $0 \%$ \\
\hline WSMO [45], [46] & 2005-2007 & From scratch & 2 & 29 & $66 \%$ \\
\hline Wedier D. Yu et al. [51] & 2007 & From scratch & 2 & 30 & $100 \%$ \\
\hline Y. Kang [52] & 2007 & From scratch & 1 & 8 & $100 \%$ \\
\hline Abramowicz et al. [20] & 2008 & Based on ISO 25000 & 2 & 17 & $100 \%$ \\
\hline N. Artaiam and T. Senivongse [53] & 2008 & Based on [16] & 2 & 12 & $89 \%$ \\
\hline S-Cube Quality Reference Model [8] & 2008 & Based on ISO/IEC 9126-1 & 3 & 66 & $100 \%$ \\
\hline onQoS [54], [55] & 2007-2009 & Based on $[26,27]$ & 3 & 25 & $74 \%$ \\
\hline BREIN QoS ontology [36], [37] & 2008-2009 & Based on $[26,28,38,50,42]$ & 3 & 40 & $67 \%$ \\
\hline Chang et al. [56] & 2009 & From scratch & 2 & 30 & $0 \%$ \\
\hline Al-Masri and Mahmoud [57], [58] & 2009 & From scratch & 3 & 19 & $41 \%$ \\
\hline Tong et al. [59] & 2009 & From scratch & 2 & 12 & $33 \%$ \\
\hline WS-QoSOnto [31], [32] & 2008-2009 & Based on [27] & 3 & 32 & $72 \%$ \\
\hline M. Comuzzi and B. Pernici [39] & 2009 & Based on $[19,16,26,28]$ & 2 & 11 & $100 \%$ \\
\hline S. Hanna et al. [33] & 2009 & Based on [49] & 1 & 8 & $100 \%$ \\
\hline Z. Balfagih and M.F. Hassan [60] & 2009 & From scratch & 3 & 26 & $91 \%$ \\
\hline Li and Zhou [61] & 2009 & From scratch & 3 & 27 & $0 \%$ \\
\hline Reddy [62] & 2009 & From scratch & 3 & 39 & $77 \%$ \\
\hline Mohanty et al. [63] & 2010 & From scratch & 1 & 10 & $90 \%$ \\
\hline Yin et al. [21], [64], [65] & 2010 & {$[27,31,42]$ and ISO/IEC 9126} & 3 & 40 & $20 \%$ \\
\hline Z. Pan and J. Baik [66] & 2010 & From scratch & 1 & 6 & $100 \%$ \\
\hline Shu and Meina [67] & 2010 & From scratch & 2 & 14 & $50 \%$ \\
\hline Lee and Yeom [68]-[72] & $2007-2010$ & From scratch & 3 & 37 & $100 \%$ \\
\hline P. Bocciarelli [73] and A. D'Ambrogio [74] & 2006-2011 & From scratch / Based on [74] & 2 & 22 & $100 \%$ \\
\hline Junping and Fan [75] & 2011 & From scratch & 1 & 8 & $100 \%$ \\
\hline Debnath et al. [76] & 2011 & From scratch & 2 & 9 & $100 \%$ \\
\hline Rosenberg et al. [77] and Moser et al. [78] & 2006-2012 & From scratch / Based on [77] & 3 & 17 & $100 \%$ \\
\hline GESSI [22]-[24] & 2008-2012 & Based on ISO/IEC 9126-1 & 3 & 57 & $98 \%$ \\
\hline WSQM [18], [19] & 2012 & Based on ISO/IEC 9126-1 & 2 & 34 & $100 \%$ \\
\hline Phalnikar and Khutade [79] & 2012 & From scratch & 2 & 20 & $0 \%$ \\
\hline Nadanam [25] & 2012 & Based on ISO/IEC 9126-1 & 3 & 34 & $100 \%$ \\
\hline Soomro and Song [44] & 2012 & Based on $[34,35]$ & 2 & 29 & $79 \%$ \\
\hline Wan Ab. Rahman et al. [80] & 2012 & From scractch / UML QoS Profile & 2 & 40 & $27 \%$ \\
\hline
\end{tabular}

$\left(^{*}\right)$ works are presented in chronological order.

\subsection{Which quality factors are the most addressed in the quality models? Which are the least addressed?}

In this question we analyze which are the most addressed quality factors of the presented quality models. To do so, we use the standard ISO/IEC 25010 [4] as a reference framework for the comparison. This standard distinguishes eight high-level characteristics, each of them 
divided into several subcharacteristics. To evaluate the coverage of the different quality characteristics and subcharacteristics on each quality model, we define the following criteria/values:

- $\quad \mathbf{Y}($ Yes): The (sub)characteristic is explicitly defined in the quality model.

- $\mathbf{Y}+($ Yes+): The (sub)characteristic is explicitly defined in the quality model and contains further subdivisions.

- $\mathbf{P}$ (Partially): The (sub)characteristic is not explicitly defined, but the quality model has a quality attribute or metric which can be classified into this (sub)characteristic.

- $\mathbf{P +}($ Partially+): The (sub)characteristic is not explicitly defined, but the quality model has several quality attributes or metrics which can be classified into this (sub)characteristic.

- ND (Not Defined): The (sub)characteristic is not defined, neither its quality attributes nor metrics.

The results of this analysis at the level of the 8 characteristics are depicted in Table 3 . As shown, the most addressed quality characteristic in quality models for web services is reliability. The importance of reliability in the field of web services is clear as most services rely on 3rd-party providers and it includes key quality attributes for selection, monitoring or adaptation.

Therefore, its dominance is not surprising. The results show that $81 \%$ of the proposals explicitly cover this characteristic and the $19 \%$ remaining, although do not define the concept explicitly, cover it partially by defining some of its subcharacteristics. In other words, there is no single proposal without addressing to some extent this characteristic.

The next most important characteristics in terms of their adoption by quality models are performance efficiency and security. These are also two key characteristics for web services, due to the fact that services operate in a highly dynamic environment over the internet.

Performance efficiency and security are explicitly covered by $68 \%$ and $74 \%$ of the proposals respectively, partially in $23 \%$ and $8 \%$, whereas proposals that do not cover them are only $9 \%$ and $17 \%$. Remarkably, only $4,3 \%$ of the proposals (i.e. 2 out of 47 ) do not cover any of them.

With respect to the rest of quality characteristics, their presence in the proposed quality models decreases dramatically. None of the remaining quality characteristics are explicitly defined by more than $20 \%$ of the proposals: maintainability is defined in $17 \%$ of the proposals, followed by usability with $10 \%$, functional suitability $4 \%$, portability $4 \%$ and compatibility $0 \%$. Although some quality models cover them partially by defining some of their subcharacteristics, their presence in quality models is very low. The clearest example is usability, which characteristic and its subcharacteristics are completely absent in $72 \%$ of the proposals. They are followed by compatibility (64\%), maintainability (64\%), portability (53\%) and functional suitability (32\%).

Table 3: ISO/IEC 25010 quality characteristics coverage in the quality models.

\begin{tabular}{|c|c|c|c|c|c|c|c|c|}
\hline & 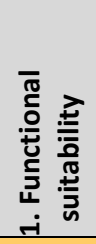 & 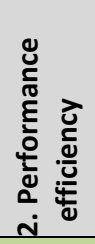 & 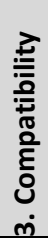 & 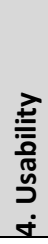 & 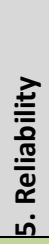 & 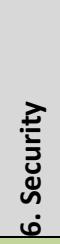 & 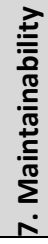 & 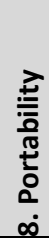 \\
\hline IBM [16] & $P$ & $Y$ & & & $\mathrm{Y}+$ & $Y$ & & \\
\hline S. $\operatorname{Ran}[26]$ & $\mathrm{P+}$ & $\mathrm{Y}+$ & & & $\mathrm{Y}+$ & $\mathrm{Y}+$ & $P$ & $P$ \\
\hline W3C QoS [28] & $P$ & $\mathrm{Y}+$ & $P$ & & $\mathrm{Y}+$ & $\mathrm{Y}+$ & & $P$ \\
\hline E. Maximilien and $\mathrm{P}$. & $P$ & $\mathrm{Y}+$ & $P$ & & $\mathrm{Y}+$ & $\mathrm{Y}+$ & $P$ & $P$ \\
\hline
\end{tabular}




\begin{tabular}{|c|c|c|c|c|c|c|c|c|}
\hline Singh [27] & & & & & & & & \\
\hline A. Avizienis et al. [34] & & & & & $\mathrm{Y}+$ & $\mathrm{Y}+$ & $\mathrm{Y}$ & \\
\hline SemWebQ [47], [48] & & $\mathrm{Y}$ & & & $\mathrm{Y}_{+}$ & $\mathrm{Y}$ & & \\
\hline Looker et al. [49] & $P$ & $\mathrm{Y}$ & & & $\mathrm{Y}+$ & $\mathrm{Y}$ & & \\
\hline QoSOnt [29] & & & & & $\mathrm{Y}+$ & $\mathrm{P}+$ & $\mathrm{Y}$ & \\
\hline WSMO-QoS [38] & $P$ & $\mathrm{Y}+$ & $P$ & & $\mathrm{Y}+$ & $\mathrm{Y}+$ & & $\mathrm{P}$ \\
\hline $\begin{array}{l}\text { S. Jiang and F. Aagesen } \\
\text { [30] }\end{array}$ & & $\mathrm{Y}$ & & & $\mathrm{Y}+$ & $\mathrm{Y}_{+}$ & & $\mathrm{P}$ \\
\hline G. Yeom el al. [40] & $P$ & $\mathrm{Y}+$ & $\mathrm{P}$ & $\mathrm{P}$ & $\mathrm{Y}+$ & $\mathrm{Y}+$ & $\mathrm{Y}_{+}$ & \\
\hline $\begin{array}{l}\text { D.T. Tsesmetzis et al. } \\
\text { [41], [42] }\end{array}$ & $P$ & $\mathrm{Y}+$ & & & $\mathrm{Y}+$ & $\mathrm{Y}_{+}$ & $P$ & $\mathrm{P}$ \\
\hline $\begin{array}{l}\text { Guimarães and Beatriz } \\
\text { [43] }\end{array}$ & $P$ & $\mathrm{P}+$ & $P$ & & $\mathrm{Y}+$ & $\mathrm{Y}$ & $P$ & $P$ \\
\hline Truong et al. [35] & $P$ & $\mathrm{Y}+$ & & & $\mathrm{Y}+$ & $\mathrm{Y}_{+}$ & & \\
\hline Ren et al. [50] & $P$ & $\mathrm{Y}+$ & & & $\mathrm{Y}+$ & $\mathrm{Y}$ & & \\
\hline WSMO [45], [46] & $P$ & $\mathrm{Y}$ & & & $\mathrm{Y}+$ & $\mathrm{Y}_{+}$ & & $\mathrm{P}$ \\
\hline Wedier D. Yu et al. [51] & $\mathrm{P}$ & $\mathrm{Y}$ & $\mathrm{P}$ & & $\mathrm{Y}+$ & $\mathrm{Y}+$ & & $\mathrm{P}$ \\
\hline Y. Kang [52] & & $Y$ & & & $\mathrm{Y}+$ & $\mathrm{Y}$ & & $\mathrm{P}$ \\
\hline Abramowicz et al. [20] & $Y$ & $Y$ & $P$ & $\mathrm{Y}$ & $Y$ & $Y$ & $Y$ & $\mathrm{Y}$ \\
\hline $\begin{array}{l}\text { N. Artaiam and T. } \\
\text { Senivongse [53] }\end{array}$ & & $\mathrm{Y}$ & & & $\mathrm{Y}+$ & $\mathrm{Y}$ & & \\
\hline $\begin{array}{l}\text { S-Cube Quality } \\
\text { Reference Model [8] }\end{array}$ & $\mathrm{P}+$ & $\mathrm{Y}+$ & & $\mathrm{P}+$ & $\mathrm{Y}+$ & $\mathrm{Y}+$ & $P$ & $P$ \\
\hline onQoS [54], [55] & & $\mathrm{Y}+$ & & & $\mathrm{Y}+$ & $\mathrm{Y}+$ & & $\mathrm{P}$ \\
\hline $\begin{array}{l}\text { BREIN QoS ontology } \\
{[36],[37]}\end{array}$ & $P$ & $\mathrm{Y}+$ & $P$ & & $\mathrm{Y}+$ & $\mathrm{Y}_{+}$ & $P$ & $\mathrm{P}$ \\
\hline Chang et al. [56] & $P$ & $\mathrm{P}+$ & $P$ & $\mathrm{Y}$ & $\mathrm{P}+$ & $\mathrm{P}+$ & & \\
\hline $\begin{array}{l}\text { Al-Masri and } \\
\text { Mahmoud [57], [58] }\end{array}$ & & $\mathrm{Y}+$ & & $\mathrm{Y}+$ & $\mathrm{Y}+$ & & & \\
\hline Tong et al. [59] & & & & & $\mathrm{Y}+$ & & & \\
\hline WS-QoSOnto [31], [32] & & $\mathrm{Y}+$ & $P$ & & $\mathrm{Y}+$ & $\mathrm{Y}+$ & & \\
\hline $\begin{array}{l}\text { M. Comuzzi and B. } \\
\text { Pernici [39] }\end{array}$ & & $P$ & & & $P$ & $P$ & & \\
\hline S. Hanna et al. [33] & $P$ & & & $\mathrm{P}+$ & $P$ & & & $\mathrm{P}+$ \\
\hline $\begin{array}{l}\text { Z. Balfagih and M.F. } \\
\text { Hassan [60] }\end{array}$ & & $P$ & $\mathrm{P}$ & $\mathrm{Y}+$ & $\mathrm{Y}+$ & $\mathrm{Y}_{+}$ & $\mathrm{Y}+$ & \\
\hline Li and Zhou [61] & $P$ & $\mathrm{Y}+$ & & & $\mathrm{Y}+$ & $\mathrm{Y}_{+}$ & & $\mathrm{P}$ \\
\hline Reddy [62] & $\mathrm{P}+$ & $\mathrm{Y}+$ & $P$ & $P$ & $P$ & $\mathrm{Y}_{+}$ & $\mathrm{Y}_{+}$ & \\
\hline Mohanty et al. [63] & $P$ & $P$ & & $P$ & $\mathrm{Y}+$ & & & \\
\hline Yin et al. [21], [64], [65] & $P$ & $\mathrm{Y}+$ & $P$ & & $\mathrm{Y}+$ & $\mathrm{Y}+$ & $P$ & $P$ \\
\hline Z. Pan and J. Baik [66] & & $\mathrm{P}+$ & & & $\mathrm{Y}+$ & & & \\
\hline Shu and Meina [67] & $P$ & $\mathrm{Y}+$ & & & $\mathrm{Y}+$ & $\mathrm{Y}+$ & & \\
\hline $\begin{array}{l}\text { Lee and Yeom [68]- } \\
{[72]}\end{array}$ & $P$ & $\mathrm{P}+$ & $P$ & $\mathrm{P}$ & $\mathrm{P}+$ & $\mathrm{Y}+$ & $\mathrm{P}+$ & \\
\hline $\begin{array}{l}\text { P. Bocciarelli [73] and } \\
\text { A. D'Ambrogio [74] }\end{array}$ & & $\mathrm{P}+$ & & & $\mathrm{Y}+$ & $P$ & & \\
\hline Junping and Fan [75] & $P$ & $\mathrm{P}+$ & & & $Y$ & $\mathrm{Y}$ & & \\
\hline Debnath et al. [76] & & $\mathrm{Y}+$ & & & $\mathrm{Y}+$ & & & \\
\hline $\begin{array}{l}\text { Rosenberg et al. [77] } \\
\text { and Moser et al. [78] }\end{array}$ & $\mathrm{P}$ & $\mathrm{Y}+$ & & & $\mathrm{P}+$ & & & $P$ \\
\hline GESSI [22]-[24] & $\mathrm{Y}_{+}$ & $\mathrm{Y}_{+}$ & $\mathrm{P}+$ & $\mathrm{Y}+$ & $\mathrm{Y}+$ & $\mathrm{Y}+$ & $\mathrm{Y}+$ & $\mathrm{Y}+$ \\
\hline
\end{tabular}




\begin{tabular}{|l|c|c|c|c|c|c|c|c|}
\hline WSQM [18], [19] & $\mathrm{P}$ & $\mathrm{P}+$ & $\mathrm{P}$ & $\mathrm{P}$ & $\mathrm{P}+$ & $\mathrm{Y}+$ & $\mathrm{Y}+$ & \\
\hline $\begin{array}{l}\text { Phalnikar and Khutade } \\
\text { [79] }\end{array}$ & $\mathrm{P}$ & $\mathrm{Y}+$ & & & $\mathrm{P}$ & $\mathrm{Y}+$ & & $\mathrm{P}$ \\
\hline Nadanam [25] & $\mathrm{P}$ & $\mathrm{P}+$ & $\mathrm{P}$ & $\mathrm{P}$ & $\mathrm{Y}+$ & & $\mathrm{P}+$ & $\mathrm{P}$ \\
\hline Soomro and Song [44] & $\mathrm{P}$ & $\mathrm{Y}+$ & & & $\mathrm{Y}+$ & $\mathrm{Y}+$ & & $\mathrm{P}$ \\
\hline $\begin{array}{l}\text { Wan Ab. Rahman et al. } \\
\text { [80] }\end{array}$ & $\mathrm{P}$ & $\mathrm{Y}+$ & & & $\mathrm{P}+$ & $\mathrm{Y}+$ & & \\
\hline $\mathrm{Y}+$ & $2 \%$ & $49 \%$ & $0 \%$ & $6 \%$ & $77 \%$ & $55 \%$ & $11 \%$ & $2 \%$ \\
\hline $\mathrm{Y}$ & $2 \%$ & $19 \%$ & $0 \%$ & $4 \%$ & $4 \%$ & $19 \%$ & $6 \%$ & $2 \%$ \\
\hline $\mathbf{P}+$ & $6 \%$ & $17 \%$ & $2 \%$ & $4 \%$ & $11 \%$ & $4 \%$ & $4 \%$ & $2 \%$ \\
\hline $\mathbf{P}$ & $57 \%$ & $6 \%$ & $34 \%$ & $13 \%$ & $8 \%$ & $4 \%$ & $15 \%$ & $40 \%$ \\
\hline (ND) & $32 \%$ & $9 \%$ & $64 \%$ & $72 \%$ & $0 \%$ & $17 \%$ & $64 \%$ & $53 \%$ \\
\hline
\end{tabular}

We describe below in further detail the completeness and coverage of subcharacteristics of each of the quality characteristics. We present here only the overall results, detailed results for each quality model can be retrieved at [12].

Functional suitability is partially covered by $63 \%$ of quality model proposals, mainly due to the functional correctness subcharacteristic (see Table 4), which includes accuracy [26], [28], [38], [78] and precision [8], [50], [56].

Table 4: Functional suitability coverage in the quality models.

\begin{tabular}{|c|c|c|c|c|}
\hline & $\begin{array}{l}\text { 1. Functional } \\
\text { suitability }\end{array}$ & $\begin{array}{l}\text { 1.1 Functional } \\
\text { completeness }\end{array}$ & $\begin{array}{l}\text { 1.2 Functional } \\
\text { correctness }\end{array}$ & $\begin{array}{l}\text { 1.3 Functional } \\
\text { appropiatenes }\end{array}$ \\
\hline $\mathrm{Y}+$ & $2 \%$ & $0 \%$ & $32 \%$ & $0 \%$ \\
\hline$Y$ & $2 \%$ & $4 \%$ & $30 \%$ & $4 \%$ \\
\hline $\mathrm{P}+$ & $6 \%$ & $0 \%$ & $2 \%$ & $0 \%$ \\
\hline P & $57 \%$ & $0 \%$ & $4 \%$ & $2 \%$ \\
\hline (ND) & $32 \%$ & $96 \%$ & $32 \%$ & $94 \%$ \\
\hline
\end{tabular}

Performance is covered by most of the quality models. However, nearly none of its subcharacteristics are explicitly defined as in ISO/IEC 25010. Instead, the quality models propose quality attributes and metrics that can be classified into these subcharacteristics, as response time, throughput and memory used. The reason for such a circumstance is that the attributes and metrics regarding performance are usually defined as direct subdivisions of the characteristic, without the subcharacteristic granularity (see Table 5). In this regard, quality attributes belonging to time behaviour are the most widespread, especially response time [26][28], [38] and throughput [16], [26]-[28].

Table 5: Performance coverage in the quality models.

\begin{tabular}{|c|c|c|c|c|}
\hline & $\begin{array}{l}\text { 2.Performance } \\
\text { efficiency }\end{array}$ & \begin{tabular}{|l}
2.1 Time \\
behaviour
\end{tabular} & $\begin{array}{l}\text { 2.2 Resource } \\
\text { utilization }\end{array}$ & 2.3 Capacity \\
\hline $\mathrm{Y}+$ & $49 \%$ & $2 \%$ & $2 \%$ & $2 \%$ \\
\hline$Y$ & $19 \%$ & $2 \%$ & $2 \%$ & $0 \%$ \\
\hline $\mathrm{P}+$ & $17 \%$ & $68 \%$ & $6 \%$ & $19 \%$ \\
\hline $\mathrm{P}$ & $6 \%$ & $15 \%$ & $6 \%$ & $45 \%$ \\
\hline (ND) & $9 \%$ & $13 \%$ & $83 \%$ & $34 \%$ \\
\hline
\end{tabular}


Compatibility is partially covered by $36 \%$ of the quality models, mainly due to the interoperability subcharacteristic. Co-existence is only present in $2 \%$ of the quality models, and no decomposition in finer-grained quality attributes is present (see Table 6).

Table 6: Compatibility coverage in the quality models.

\begin{tabular}{|c|r||r|r|}
\hline & 3. Compatibility & \multicolumn{3}{|l|}{3.1 Co-existence } & \multicolumn{2}{l|}{$\begin{array}{l}\text { l.2 Interopera- } \\
\text { bility }\end{array}$} \\
\hline$Y+$ & $0 \%$ & $0 \%$ & $2 \%$ \\
\hline$Y$ & $0 \%$ & $2 \%$ & $34 \%$ \\
\hline$P+$ & $2 \%$ & $0 \%$ & $0 \%$ \\
\hline$P$ & $34 \%$ & $0 \%$ \\
\hline (ND) & $64 \%$ & $98 \%$ & $64 \%$ \\
\hline
\end{tabular}

Usability is mostly ignored in the quality models. It is explicitly defined only in $10 \%$ of the quality models and partially covered in $17 \%$ of quality models. This partial coverage is due to the appropriate recognizability, operability and learnability subcharacteristics. Appropriate recognizability includes the quality attributes documentation [33], [57], [63] and discoverability [60]. Operability includes, as the main quality attribute, controllability [18], [19], [40]. On the contrary, learnability has no decomposition (see Table 7).

Table 7: Usability coverage in the quality models.

\begin{tabular}{|c|c|c|c|c|c|c|c|}
\hline & 4. Usability & $\begin{array}{l}\text { 4.1 Appropriate } \\
\text { recognizability }\end{array}$ & 4.2 Learnability & 4.3 Operability & $\begin{array}{l}4.4 \text { User error } \\
\text { protection }\end{array}$ & $\begin{array}{l}\text { 4. } 5 \text { User interfa- } \\
\text { ce aesthetics }\end{array}$ & $\begin{array}{l}\text { 4.6 Accessi- } \\
\text { bility }\end{array}$ \\
\hline$Y_{+}$ & $6 \%$ & $6 \%$ & $0 \%$ & $4 \%$ & $0 \%$ & $0 \%$ & $0 \%$ \\
\hline$Y$ & $4 \%$ & $2 \%$ & $4 \%$ & $2 \%$ & $0 \%$ & $0 \%$ & $0 \%$ \\
\hline $\mathrm{P}+$ & $4 \%$ & $0 \%$ & $0 \%$ & $0 \%$ & $0 \%$ & $0 \%$ & $0 \%$ \\
\hline $\mathrm{P}$ & $13 \%$ & $6 \%$ & $0 \%$ & $2 \%$ & $0 \%$ & $0 \%$ & $0 \%$ \\
\hline (ND) & $72 \%$ & $85 \%$ & $95 \%$ & $91 \%$ & $100 \%$ & $100 \%$ & $100 \%$ \\
\hline
\end{tabular}

Reliability is the most covered characteristic by existing quality models. Regarding its subcharacteristics, the most covered is availability, which is remarkably defined in $94 \%$ of the proposed quality models. The other subcharacteristics have also some arguably good coverage with the exception of maturity which is only covered in $2 \%$ and partially covered in $11 \%$ of quality models (see Table 8 ). The partial coverage of maturity comes from the consistency quality attribute [21], [27], [31], [36], [37].

Table 8: Reliability coverage in the quality models.

\begin{tabular}{|c|c|c|c|c|c|}
\hline & 5. Reliability & 5.1 Maturity & 5.2 Availability & $\begin{array}{l}5.3 \text { Fault } \\
\text { tolerance }\end{array}$ & $\begin{array}{l}\text { 5.4 Recovera- } \\
\text { bility }\end{array}$ \\
\hline $\mathrm{Y}+$ & $77 \%$ & $0 \%$ & $28 \%$ & $13 \%$ & $23 \%$ \\
\hline$Y$ & $4 \%$ & $2 \%$ & $66 \%$ & $26 \%$ & $6 \%$ \\
\hline $\mathrm{P}+$ & $11 \%$ & $0 \%$ & $0 \%$ & $0 \%$ & $2 \%$ \\
\hline $\mathrm{P}$ & $9 \%$ & $11 \%$ & $0 \%$ & $0 \%$ & $13 \%$ \\
\hline (ND) & $0 \%$ & $87 \%$ & $6 \%$ & $62 \%$ & $55 \%$ \\
\hline
\end{tabular}

Security is explicitly defined by $74 \%$ of quality models. Regarding its subcharacteristics, all of them have been explicitly defined with similar percentages, which shows a clear consolidation 
on its decomposition. The main difference appears in confidentiality (predominance of $Y+$ over Y) because most quality models present an encryption-related quality attribute [18], [21], [27], [31] which is a concept that clearly falls into it (see Table 9).

Table 9: Security coverage in the quality models.

\begin{tabular}{|c|c|c|c|c|c|c|}
\hline & 6. Security & $\begin{array}{l}6.1 \text { Confiden- } \\
\text { tiality }\end{array}$ & 6.2 Integrity & $\begin{array}{l}6.3 \text { Non- } \\
\text { repudiation }\end{array}$ & $\begin{array}{l}6.4 \text { Accounta- } \\
\text { bility }\end{array}$ & 6.5 Authenticity \\
\hline $\mathrm{Y}+$ & $55 \%$ & $36 \%$ & $0 \%$ & $0 \%$ & $19 \%$ & $0 \%$ \\
\hline $\mathrm{Y}$ & $19 \%$ & $9 \%$ & $43 \%$ & $43 \%$ & $28 \%$ & $45 \%$ \\
\hline $\mathrm{P}+$ & $4 \%$ & $4 \%$ & $0 \%$ & $0 \%$ & $0 \%$ & $0 \%$ \\
\hline P & $4 \%$ & $11 \%$ & $0 \%$ & $0 \%$ & $0 \%$ & $0 \%$ \\
\hline (ND) & $17 \%$ & $40 \%$ & $57 \%$ & $57 \%$ & $53 \%$ & $55 \%$ \\
\hline
\end{tabular}

Maintainability is only explicitly defined in $17 \%$ of the quality models, and partially supported in $19 \%$. The partial coverage is mainly due to the modifiability subcharacteristic which is also partially covered by the stability/change cycle quality attribute [8], [26], [27], [41] (see Table 10).

Table 10: Maintainability coverage in the quality models.

\begin{tabular}{|c|c|c|c|c|c|c|}
\hline & $\begin{array}{l}\text { 7. Maintaina- } \\
\text { bility }\end{array}$ & 7.1 Modularity & 7.2 Reusability & $\begin{array}{l}\text { 7.3 Analysa- } \\
\text { bility }\end{array}$ & $\begin{array}{l}7.4 \text { Modifia- } \\
\text { bility }\end{array}$ & 7.5 Testability \\
\hline $\mathrm{Y}+$ & $11 \%$ & $0 \%$ & $2 \%$ & $0 \%$ & $2 \%$ & $0 \%$ \\
\hline $\mathrm{Y}$ & $6 \%$ & $11 \%$ & $2 \%$ & $2 \%$ & $0 \%$ & $2 \%$ \\
\hline $\mathrm{P}+$ & $4 \%$ & $0 \%$ & $0 \%$ & $9 \%$ & $4 \%$ & $0 \%$ \\
\hline $\mathrm{P}$ & $15 \%$ & $0 \%$ & $0 \%$ & $0 \%$ & $13 \%$ & $0 \%$ \\
\hline (ND) & $64 \%$ & $89 \%$ & $96 \%$ & $89 \%$ & $81 \%$ & $98 \%$ \\
\hline
\end{tabular}

Portability is partially covered in nearly half of the presented quality models due to the adaptability subcharacteristic, which is partially covered by the scalability quality attribute that appears in a few proposals [26]-[28], [38] (see Table 11).

Table 11: Portability coverage in the quality models.

\begin{tabular}{|c|c|c|c|c|}
\hline & 8. Portability & 8.1 Adaptability & 8.2 Installability & $\begin{array}{l}8.3 \text { Replacea- } \\
\text { bility }\end{array}$ \\
\hline $\mathrm{Y}+$ & $2 \%$ & $2 \%$ & $0 \%$ & $0 \%$ \\
\hline$Y$ & $2 \%$ & $2 \%$ & $2 \%$ & $4 \%$ \\
\hline $\mathrm{P}+$ & $2 \%$ & $0 \%$ & $0 \%$ & 09 \\
\hline $\mathrm{P}$ & $40 \%$ & $40 \%$ & $0 \%$ & $0 \%$ \\
\hline (ND) & $53 \%$ & $55 \%$ & $98 \%$ & 96 \\
\hline
\end{tabular}

Non-technical characteristics are not present in ISO25010, because the standard focuses on technical aspects of the software. Nevertheless, since they represent an important aspect when evaluating web services for their prospective use, we have also considered them. We have taken as the basis for comparison the extension of ISO-9126 with non-technical quality characteristics presented in [81]. We adapted the subcharacteristics to the context of web services, therefore 
the non-technical subcharacteristics we propose are regulatory (i.e. related to laws and standards), economic (i.e. related to costs and penalties) and reputation and recognition. Analyzing the results, economic is the most used non-technical subcharacteristic, being cost its main quality attribute [26], [30], [38], [47], [48] (see Table 12).

Table 12: Non-technical coverage in the quality models.

\begin{tabular}{|c|c|c|c|c|}
\hline & 9. Non-technical & 9.1 Regulatory & 9.2 Economic & $\begin{array}{l}\text { 9.3 Reputation } \\
\text { \& Recognition }\end{array}$ \\
\hline $\mathrm{Y}+$ & $2 \%$ & $4 \%$ & $17 \%$ & $4 \%$ \\
\hline$Y$ & $0 \%$ & $11 \%$ & $0 \%$ & $0 \%$ \\
\hline $\mathrm{P}+$ & $43 \%$ & $13 \%$ & $13 \%$ & $9 \%$ \\
\hline $\mathrm{P}$ & $30 \%$ & $9 \%$ & $30 \%$ & $26 \%$ \\
\hline (ND) & $26 \%$ & $64 \%$ & $40 \%$ & $62 \%$ \\
\hline
\end{tabular}

Some relevant observations follow:

Unbalance between client-based and provider-based quality attributes: Client-based quality attributes are those which are measured from the client's perspective, whereas provider-based quality attributes are those measured from the provider's perspective. It is worth to remark the unbalance between client-based and provider-based quality attributes, being the latter less covered. For instance, response time (which is from the client's perspective) is present in $83 \%$ of the quality models, whereas execution time (which it is from the provider's perspective) is present in $23 \%$ of quality models. This has an impact on the coverage of certain subcharacteristics. For instance resource utilization (which clearly deals with the provider's perspective) is only partially covered in $17 \%$ of the quality models whereas time behaviour is covered in $87 \%$.

Lack of some subcharacteristics support: We have identified some ISO/IEC 25010 quality subcharacteristics that, to our understanding, have not been addressed enough in current quality models. For instance, the lack of the modularity and reusability subcharacteristics contrasts with the fact that they are two key principles in service oriented architecture [82]. On the other hand, appropriate recognizability and functional appropriateness are remarkably subcharacteristics for a proper web service discovery that have not been widely addressed. Similarly, other relevant characteristics that have not been much addressed are the usability and maintainability subcharacteristics. We argue that some of these results may be due to the unbalance between client-based and provider-based quality attributes.

Terminological inconsistencies with the standard: We found some terminological inconsistencies with the standard ISO25010. It is remarkable that many quality models have a quality attribute named accessibility, but they refer to a completely different concept. In most quality models, it refers to the availability of the system under certain circumstances (e.g. limit of concurrent users) [16], [28], [38], [47], [48] whereas in ISO25010 it refers to the accessibility for people with disabilities. Also some quality models have a quality attribute named capacity, but its definition is limited to the maximum throughput [26]-[28], [38], which is a subset of the definition of capacity in ISO25010.

\subsection{What are the most consolidated quality factors?}


In this question we aim at identifying the most consolidated definitions for quality factors in the surveyed quality models. We restrict the analysis to those quality attributes that have appeared most frequently in the surveyed quality models. We have determined a threshold value of $30 \%$ (i.e. we are considering those quality attributes that appear at least in $30 \%$ of the surveyed quality models). Such threshold value is arbitrary as we found no procedure in the literature to define it. Nevertheless, after several iterations, we considered it an appropriate value since it yielded as result 19 quality attributes, which is arguably a reasonable number for this kind of analysis. Furthermore, we observed that for most of quality attributes below this threshold, the number of terminology discrepancies is higher (details are presented in [12]). On the other hand, a higher threshold can be easily analysed since the usage percentage is shown in Table 13.

In order to obtain accurate and significant results, the analysis was based on the definition of the quality attributes. That is, we established a mapping between the different quality attributes that shared the same or nearly equivalent definitions. Nevertheless, as we need to use a name for each of them, we use the term that is more used along the different quality models. The list of terms and definitions is depicted in Table 13.

In the first column, we depict the quality attributes classified into the ISO/IEC 25010 quality subcharacteristic. In the second column, we provide the most consolidated definition. The percentage of quality models that have such quality attribute based on its definition (i.e. quality models that have another name for such quality attribute do also count in the percentage) is shown in the third column. Finally, from the quality models that have such quality attribute based on the definition, we depict the percentage of them that use the most common name.

For readability, the subcharacteristics are grouped into quality characteristics (functional suitability, etc.), see rows in grey in the table. 
Table 13: Most consolidated quality attributes and their definitions.

\begin{tabular}{|c|c|c|c|}
\hline $\begin{array}{l}\text { ISO/IEC } 25010 \Rightarrow \text { Quality } \\
\text { subcharacteristics attribute }\end{array}$ & Definition & Definition usage \% & Name usage \% \\
\hline \multicolumn{4}{|c|}{ Functional suitability } \\
\hline $\begin{array}{l}\text { Functional } \Rightarrow \text { Accuracy } \\
\text { correctness }\end{array}$ & Error rate produced by the service $[26][28]$. & $62 \%$ & $42 \%$ \\
\hline \multicolumn{4}{|c|}{ Performance efficiency } \\
\hline Capacity $\Rightarrow$ Accessibility & $\begin{array}{l}\text { Degree the service is capable of serving a web } \\
\text { service request [53][16]. }\end{array}$ & $47 \%$ & $100 \%$ \\
\hline Capacity $\Rightarrow$ Capacity $^{1}$ & $\begin{array}{l}\text { Limit of concurrent requests for guaranteed } \\
\text { performance [26][55]. }\end{array}$ & $51 \%$ & $65 \%$ \\
\hline Time behaviour $\Rightarrow$ Response Time & $\begin{array}{l}\text { Time to complete a WS request (from a client } \\
\text { perspective) [26][28]. }\end{array}$ & $8 \beta \%$ & $73 \%$ \\
\hline Time behaviour $\Rightarrow$ Throughput & $\begin{array}{l}\text { Number of web service requests served at a given } \\
\text { time period [28][16]. }\end{array}$ & $60 \%$ & $100 \%$ \\
\hline \multicolumn{4}{|c|}{ Compatibility } \\
\hline (subcharacteristic) Interoperability & $\begin{array}{l}\text { Ease with which a consumer application or agent } \\
\text { interoperates with a service [27]. }\end{array}$ & $36 \%$ & $100 \%$ \\
\hline \multicolumn{4}{|c|}{ Reliability } \\
\hline Availability $\Rightarrow$ Availability $^{1}$ & $\begin{array}{l}\text { Probability that the service can respond to } \\
\text { consumer requests [51][27]. }\end{array}$ & $94 \%$ & $98 \%$ \\
\hline Recoverability $\Rightarrow$ MTTR & Mean Time to Repair [30][27]. & $30 \%$ & \\
\hline Fault tolerance $\Rightarrow$ Robustness & $\begin{array}{l}\text { Degree to which a service can function correctly in } \\
\text { the presence of invalid, incomplete or conflicting } \\
\text { inputs }[26][28] \text {. }\end{array}$ & $38 \%$ & 94 \\
\hline \multicolumn{4}{|c|}{ Security } \\
\hline (subcharacteristic) Authentication & $\begin{array}{l}\text { Measure of how the service authenticates } \\
\text { principals (users or other services) who can access } \\
\text { service and data [26]. }\end{array}$ & $45 \%$ & $95 \%$ \\
\hline (subcharacteristic) Confidentiality & $\begin{array}{l}\text { Measure of how the service treats the data, so } \\
\text { that only authorized principals can access or } \\
\text { modify the data [26]. }\end{array}$ & $45 \%$ & $86 \%$ \\
\hline (subcharacteristic) Integrity & $\begin{array}{l}\text { Absence of improper system state alterations } \\
\text { including accidental or malicious alternation or } \\
\text { removal of information [8][29]. }\end{array}$ & $43 \%$ & $100 \%$ \\
\hline (subcharacteristic) $\begin{array}{l}\text { Non- } \\
\text { repudiation }\end{array}$ & $\begin{array}{l}\text { A principal cannot deny requesting a service or } \\
\text { data after the fact [26][28]. }\end{array}$ & $43 \%$ & $100 \%$ \\
\hline Accountability $\Rightarrow \begin{array}{l}\text { Traceability and } \\
\text { auditability }\end{array}$ & $\begin{array}{l}\text { Whether it is possible to trace the history of a } \\
\text { service when a request was serviced [26]. }\end{array}$ & $38 \%$ & $95 \%$ \\
\hline Confidentiality $\Rightarrow$ Authorization & $\begin{array}{l}\text { Measure of how the service authorizes principals } \\
\text { so that only them can access the protected } \\
\text { services [26]. }\end{array}$ & $40 \%$ & $67 \%$ \\
\hline Confidentiality $\Rightarrow$ Encryption & $\begin{array}{l}\text { Measure of how the service encrypts data. Type } \\
\text { and strength of encryption technology used for } \\
\text { storage and messaging [26]. }\end{array}$ & $40 \%$ & $100 \%$ \\
\hline \multicolumn{4}{|c|}{ Portability } \\
\hline Adaptability $\Rightarrow$ Scalability & $\begin{array}{l}\text { Capability of increasing the computing capacity of } \\
\text { service provider's system and system's ability to } \\
\text { process more operations in a given period[26][8]. }\end{array}$ & $45 \%$ & $100 \%$ \\
\hline \multicolumn{4}{|c|}{ Non-technical } \\
\hline Economic $\Rightarrow$ Cost & $\begin{array}{l}\text { Measure of the cost involved in requesting the } \\
\text { service [26][52]. }\end{array}$ & $60 \%$ & $55 \%$ \\
\hline $\begin{array}{l}\text { Reputation } \Rightarrow \text { Reputation } \\
\text { and recognition }\end{array}$ & $\begin{array}{l}\text { It is a measure of service trustworthiness. It } \\
\text { depends on end user's experiences of using the } \\
\text { service [8][40]. }\end{array}$ & $38 \%$ & $73 \%$ \\
\hline
\end{tabular}

(1): The quality attribute has the same name as the ISO subcharacteristic, but it refers to a different concept, as the scope of its definition is more delimited 
With respect to the list of quality attributes, it is worth to remark two issues that we have identified:

- The interoperability and security subcharacteristics are considered quality attributes in most quality models, although they are defined as subcharacteristics in ISO/IEC 25010.

- Similarly, capacity and availability are defined as quality attributes in most quality models, whereas in ISO/IEC 25010, the same terms are considered subcharacteristics. Nevertheless, in these cases, the definitions in the proposed quality models do not refer exactly to the same concept as in ISO/IEC 25010. Availability, in most quality models, refer to the probability that the service can respond to consumer requests [27], [51], whereas in ISO/IEC 25010 it is a subcharacteristics that may include other quality attributes to measure the degree to which the service is operational (e.g. total uptime). Similarly, capacity, as defined in the proposed quality models, is limited to the maximum throughput [26]-[28], [38].

Regarding the results of the analysis, the most widespread quality attribute we found is by far availability, which is used in $94 \%$ of the quality models, followed by response time ( $83 \%)$, accuracy (62\%), throughput (60\%) and cost (60\%).

Regarding their names, there is a wide consensus for the terms to use over these quality attributes. All of them have a common terminology in the majority (i.e. more than a half) of the quality models. Moreover, most of these quality attributes share the same name in over $90 \%$ of the quality models. Nevertheless we can distinguish some discrepancies on the terms to use in the following quality attributes: accuracy (other names are, e.g. error rate [21], [38], successability [18], [40]), capacity (e.g. maximum throughput [40], [56]), response time (e.g. latency [16], [49]), authorization (e.g. service access control [73], [74]), confidentiality (e.g. privacy [18], [56]), cost (e.g. price [18], [57]) and reputation (e.g. trust [21], [46]).

\section{Threats to validity}

In this section we discuss all the aspects during the research process that might lead to a threat to validity, as well as the actions we have performed in order to mitigate those risks. In this regard, we identified and evaluated the threats following the common classification of construct validity, internal validity, external validity and conclusion validity.

Construct validity: Construct validity refers to the validity threats with respect to the observations performed in the study and if they really represent what is being investigated. At this respect, one of the inherent threats to any systematic mapping is that it does not guarantee the inclusion of all the relevant works in the field. This might be caused by several reasons, for instance, a relevant work may not be indexed on the selected database, the keywords used in the title or abstract of a relevant work do not match with the keywords of the search, etc. This threat was mitigated by combining several databases (ISI WOS, IEEE Xplore and ACM DL) and manual searches to selected journals and conferences, as well as studying accurately the keywords to use. However, the issue may not be solved since the problem goes beyond an accurate protocol and concerns also issues related to the paper (e.g. inaccurate abstracts). To mitigate this risk, we included a final step of snowballing, as described in Section 3.3.3, assuring that the quality models that have had the biggest impact in the field were also included. Also, the identification of some basic sources was helpful since, as summarized in Table 1, some of the 
conferences that we considered as the usual venues for the topic of the systematic mapping, had at least one edition not indexed in the selected databases, but still we handled them manually.

Internal validity: Internal validity refers to the validity of the analysis performed. Concerning this aspect, we have identified two major threats.

(1) Not all the quality models define all the presented quality factors accurately. As shown in Section 4.2, some definitions are ambiguous, inconsistent or simply absent. This situation poses a challenge when analyzing the coverage of the characteristics and subcharacteristics, and a subsequent threat to validity. To overcome such threat, we examined the list of not-well defined quality factors and took the following strategy: for those factors lacking of a definition, if there was a clear consensus of the definition in the state of the art, or the name of the quality factor was self-explanatory (e.g. total memory consumption [36]), we recognized the meaning of the quality factor despite the lack of definition. For those factors whose definition was ambiguous or inconsistent, if such ambiguity or inconsistency didn't affect the categorization of the quality factor, we included that quality factor in the analysis. Otherwise, the quality factor was discarded from the analysis. Following this criterion, 35 quality factors were discarded from more than 1000 quality factors analysed $(<3,5 \%)$.

(2) As each quality model implements its own hierarchical structure, we decided to map these structures into a reference quality model structure. To this aim, we used the standard ISO/IEC 25010 as it is described in [4], and decided the mapping of the nodes from each quality model to this standard. This mapping can be considered by itself a threat to validity. To mitigate this risk, the mapping was discussed and analysed closely by all three authors of the paper. Nevertheless, some nodes could not be clearly mapped into a subcharacteristic of ISO/IEC 25010. This is issue is present only in just 14 nodes out of $134(<10,5 \%)$.

External validity: External validity is concerned about the extrapolation of the results from a particular scenario to the general case. Since our results are scoped in quality models in web services and we do not attempt to generalize conclusions beyond this scope, this validity threat does not apply.

Conclusion validity: Conclusion validity is concerned about whether the research performed is reproducible by other researchers with similar results. In this regard, we have explicitly described all the steps performed in the systematic mapping by detailing the procedure as defined in the systematic mapping protocol. Furthermore, the list of papers found and selected on each step is included in [12].

\section{Conclusions}

In this systematic literature review we have surveyed the state of the art in the definition of quality models for web services. The interest on quality models stems from their applicability in the study of the wider concept of quality of service. We designed and followed a rigorous protocol which uncovered up to 65 papers presenting 47 proposals to answer the different research questions that we identified. We may consider the answer to these questions as the main outcome of this paper. We summarize these answers below:

\section{$R Q$ 1.1. What is the chronological overview of the research done so far in quality models for} web services? 
Quality models for web services have been an increasingly addressed research topic from 2001 to the current days, with the exception of the last 2-year period. We have distributed chronologically the 47 proposals showing their relationships and identifying which ones are the most consolidated ones, the most influencing ones and the most influenced ones.

\section{$R Q$ 1.2. What are the characteristics of the proposed quality models?}

We have observed that the size varies among 6 and 66 nodes (with an average of 24,38) and the number of levels among 1 and 3 (with an average of 2,23), with a correlation between both factors. We have also observed that a narrow majority of proposals (51\%) have a unique and consistent definition for all their quality factors, and only $19 \%$ of them have a completeness definition below $40 \%$.

\section{$R Q$ 1.3. Which quality factors are the most addressed in the quality models? Which are the least addressed?}

Our study has shown that first reliability and then security and performance efficiency are the ISO/IEC 25010 characteristics explicitly defined in at least half of the surveyed proposals. Concerning subcharacteristics, the ones explicitly or defined in at least half of the proposals are: functional correctness and availability. Also, time behaviour, capacity, confidentiality and economy exceed this threshold if implicit definitions are considered too.

\section{$R Q$ 1.4. What are the most consolidated quality factors?}

We have obtained up to 19 quality attributes that appear in at least $30 \%$ of the surveyed quality models. Among them, availability is the most used and has an almost universally-agreed definition; also response time, throughput, cost and accuracy appear in more than half of the proposals. In general, we can say that there is much consensus in the definitions of these 19 most popular quality attributes: up to 7 of them are defined the same way in all the quality models that introduce them, and the rest of them are still consistently used in more than a half of the proposals, with the exception of accuracy.

As a final conclusion, we believe that this panoramic view on the anatomy of quality models for web services may be a good reference for prospective researchers and practitioners on the field, especially with the aim of avoiding new classifications or definitions that may be contrary to the established practices that we have found.

As future work, we plan to investigate in depth the semantical representation of the quality factors in existing web service ontologies. Specifically, how current web service ontologies in the literature support the instantiation of the quality models and their quality factors in an ontological model for a proper reasoning and analysis on QoS.

\section{References}

[1] G. Alonso, F. Casati, H. Kuno, and V. Machiraju, Web services. Springer, 2004.

[2] Y. Wei and Mb. Blake, "Service-oriented computing and cloud computing: Challenges and opportunities," Internet Comput. IEEE, vol. 14, no. 6, pp. 72-75, 2010.

[3] B. Kitchenham and S. Charters, "Guidelines for performing systematic literature reviews in software engineering, Version 2.3," 2007. 
[4] ISO/IEC, "ISO/IEC 25010 - Systems and software engineering - Systems and software Quality Requirements and Evaluation (SQuaRE) - System and software quality models," ISO/IEC, 2010.

[5] Institute of Electrical and Electronics, "IEEE 610.12-1990: IEEE Standard Glossary of Software Engineering Terminology." 1990.

[6] ISO IEC, "ISO 8402:1994 - Quality management and quality assurance - Vocabulary." 1994.

[7] I. Burnstein, Practical software testing: a process-oriented approach. Springer, 2003.

[8] A. Gehlert and A. Metzger (eds.), "CD-JRA-1.3.2 Quality Reference Model for SBA,” 2009.

[9] I. J. Jureta, C. Herssens, and S. Faulkner, "A comprehensive quality model for serviceoriented systems," Softw. Qual. J., vol. 17, no. 1, pp. 65-98, 2009.

[10] B. Kitchenham, R. Pretorius, D. Budgen, P. Brereton, M. Turner, M. Niazi, and S. G. Linkman, "Systematic literature reviews in software engineering - A tertiary study," Inf. Softw. Technol., vol. 52, no. 8, pp. 792-805, 2010.

[11] K. Petersen, R. Feldt, S. Mujtaba, and M. Mattsson, "Systematic mapping studies in software engineering," in Proceedings of the 12th international conference on Evaluation and Assessment in Software Engineering (EASE), 2008, pp. 68-77.

[12] M. Oriol, X. Franch, and J. Marco, "Appendix of: quality models for web services, a systematic mapping," 2013. [Online]. Available: http://gessi.Isi.upc.edu/qmodels/.

[13] B. Kitchenham, P. Brereton, M. Turner, M. Niazi, S. Linkman, R. Pretorius, and D. Budgen, "The impact of limited search procedures for systematic literature reviews A participantobserver case study," in Proceedings of the 2009 3rd International Symposium on Empirical Software Engineering and Measurement, 2009, pp. 336-345.

[14] B. A. Kitchenham, E. Mendes, and G. H. Travassos, "Cross versus Within-Company Cost Estimation Studies: A Systematic Review," IEEE Trans.Software Eng., vol. 33, no. 5, pp. 316-329, 2007.

[15] M. Riaz, E. Mendes, and E. Tempero, "A systematic review of software maintainability prediction and metrics," in Proceedings of the 2009 3rd International Symposium on Empirical Software Engineering and Measurement, 2009, pp. 367-377.

[16] A. Mani and A. Nagarajan, "Understanding quality of service for Web services," 2002. [Online]. Available: http://www-106.ibm.com/developerworks/library/ws-quality.html.

[17] ISO IEC, "ISO 9126/ISO, IEC (Hrsg.): International Standard ISO/IEC 9126: Information Technology-Software Product Evaluation." 1991.

[18] OASIS, "Web Services Quality Factors Version 1.0," 2012. [Online]. Available: http://docs.oasis-open.org/wsqm/wsqf/v1.0/WS-Quality-Factors.pdf. 
[19] OASIS, "OASIS WS quality model TC - Quality model for Web services.," 2005. [Online]. Available: http://www.oasis-open.org/committees/tc \home.php?wg Łabbrev=wsqm.

[20] W. Abramowicz, R. Hofman, W. Suryn, and D. Zyskowski, "SQuaRE based Web Services Quality Model," in Proceedings of the International MultiConference of Engineers and Computer Scientists 2008, Vol I IMECS, 2008, pp. 827-835.

[21] B. Yin, H. Yang, P. Fu, and X. Chen, "A semantic web services discovery algorithm based on QoS ontology," in Proceedings of the 6th international conference on Active media technology, 2010, pp. 166-173.

[22] D. Ameller and X. Franch, "Service Level Agreement Monitor (SALMon)," in Proceedings of the Seventh International Conference on Composition-Based Software Systems (ICCBSS), 2008, pp. 224-227.

[23] M. Oriol, J. Marco, X. Franch, and D. Ameller, "Monitoring Adaptable SOA-Systems using SALMon," in Workshop on Service Monitoring, Adaptation and Beyond (Mona+), 2008, pp. 19-28.

[24] O. Cabrera and X. Franch, "A quality model for analysing web service monitoring tools," in Sixth International Conference on Research Challenges in Information Science (RCIS), 2012, pp. 1-12.

[25] P. Nadanam and R. Rajmohan, "QoS evaluation for web services in cloud computing," in Third International Conference on Computing, Communication and Networking Technologies (ICCCNT), 2012, pp. 1-8.

[26] S. Ran, "A model for web services discovery with QoS," SIGecom Exch., vol. 4, no. 1, pp. 1-10, 2003.

[27] E. M. Maximilien and M. P. Singh, "A framework and ontology for dynamic Web services selection," Internet Comput. IEEE, vol. 8, no. 5, pp. 84-93, 2004.

[28] K. Lee, J. Jeon, W. Lee, S. Jeong, and S. Park, "QoS for Web Services: Requirements and Possible Approaches," W3C Working group Note 25, 2003. [Online]. Available: http://www.w3c.or.kr/kr-office/TR/2003/ws-qos/.

[29] G. Dobson, R. Lock, and I. Sommerville, "QoSOnt: a QoS ontology for service-centric systems," in Proceedings of the 31st EUROMICRO Conference on Software Engineering and Advanced Applications, 2005, pp. 80-87.

[30] S. Jiang and F. Aagesen, "An Approach to Integrated Semantic Service Discovery," in Autonomic Networking, Springer Berlin Heidelberg, 2006, pp. 159-171.

[31] V. X. Tran, H. Tsuji, and R. Masuda, "A new QoS ontology and its QoS-based ranking algorithm for Web services," Dependable Serv. Comput. Syst., vol. 17, no. 8, pp. 13781398, 2009.

[32] V. X. Tran, "WS-QoSOnto: A QoS Ontology for Web Services," in IEEE International Symposium on Service-Oriented System Engineering (SOSE), 2008, pp. 233-238. 
[33] S. Hanna and A. Alalawneh, "An Ontology for the Quality Attributes of Web Services," in 13th IBIMA Conference, 2009.

[34] A. Avizienis, J.-C. Laprie, B. Randell, and C. Landwehr, "Basic concepts and taxonomy of dependable and secure computing," Dependable Secur. Comput. IEEE Trans., vol. 1, no. 1, pp. 11-33, 2004.

[35] H. Truong, R. Samborski, and T. Fahringer, "Towards a Framework for Monitoring and Analyzing QoS Metrics of Grid Services," in 2006 Second IEEE International Conference on e-Science and Grid Computing (e-Science'06), 2006, pp. 65-65.

[36] H. Muñoz Frutos, I. Kotsiopoulos, L. M. Vaquero Gonzalez, and L. Rodero Merino, "Enhancing Service Selection by Semantic QoS," in Proceedings of the 6th European Semantic Web Conference on The Semantic Web: Research and Applications, 2009, vol. Heraklion,, pp. 565-577.

[37] H. Muñoz Frutos, A. Micsik, D. Fellows, J. Leukel, R. Woitsch, H. Eichner, B. Cantalupo, and A. Giuseppe, "Final Report on BREIN-Core Ontologies D3.2.5," 2008. [Online]. Available: http://www.eubrein.com/index.php?option=com_docman\&task=doc_view\&gid=57.

[38] X. Wang, T. Vitvar, M. Kerrigan, and I. Toma, "A qos-aware selection model for semantic web services," in Proceedings of the 4th international conference on Service-Oriented Computing, 2006, pp. 390-401.

[39] M. Comuzzi and B. Pernici, "A framework for QoS-based Web service contracting," ACM Trans.Web, vol. 3, no. 3, pp. 10:1-10:52, 2009.

[40] G. Yeom, T. Yun, and D. Min, "QoS Model and Testing Mechanism for Quality-driven Web Services Selection," in Proceedings of the The Fourth IEEE Workshop on Software Technologies for Future Embedded and Ubiquitous Systems, and the Second International Workshop on Collaborative Computing, Integration, and Assurance (SEUS-WCCIA'06), 2006, pp. 199-204.

[41] D. T. Tsesmetzis, I. G. Roussaki, I. V Papaioannou, and M. E. Anagnostou, "QoS awareness support in Web-Service semantics," in International Conference on Internet and Web Applications and Services/Advanced International Conference onTelecommunications. AICT-ICIW., 2006, p. 128.

[42] I. V Papaioannou, D. T. Tsesmetzis, I. G. Roussaki, and M. E. Anagnostou, "A qos ontology language for web-services," in Proceedings of the 20th International Conference on Advanced Information Networking and Applications, 2006.

[43] D. Z. G. Garcia and M. B. F. de Toledo, "Semantics-enriched QoS policies for web service interactions," in Proceedings of the 12th Brazilian Symposium on Multimedia and the web, 2006, pp. 35-44.

[44] A. Soomro and W. Song, "Developing and Managing SLAs for Business Applications in Information Systems," in Emerging Trends and Applications in Information Communication Technologies, 2012, pp. 489-500. 
[45] J. Brujin, C. Bussler, J. Domingue, D. Fensel, M. Hepp, M. Kifer, B. König-Ries, J. Kopecky, R. Lara, E. Oren, A. Polleres, J. Scicluna, and M. Stollberg, "D2v1.4. Web Service Modeling Ontology (WSMO)," WSMO Working Draft, 2007. [Online]. Available:

http://www.wsmo.org/TR/d2/v1.4/.

[46] J. Brujin, C. Bussler, J. Domingue, D. Fensel, M. Hepp, U. Keller, M. Kifer, B. König-Ries, J. Kopecky, R. Lara, H. Lausen, E. Oren, A. Polleres, D. Roman, J. Scicluna, and M. Stollberg, "Web service modeling ontology (WSMO)," W3C Member Submission, 2005. .

[47] C. Patel, K. Supekar, and Y. Lee, "Provisioning resilient, adaptive Web services-based workflow: a semantic modeling approach," in Proceedings of IEEE International Conference on Web Services, 2004, pp. 480-487.

[48] C. Patel, K. Supekar, and Y. Lee, "A QoS Oriented Framework for Adaptive Management of Web Service based Workflows," in In Proceeding of Database and Expert Systems Conference., 2003, pp. 826-835.

[49] N. Looker, M. Munro, and J. Xu, "Assessing Web Service Quality of Service with Fault Injection," in Workshop on Quality of Service for Application Servers, 2004.

[50] K. Ren, J. Chen, T. Chen, J. Song, and N. Xiao, "Grid-Based Semantic Web Service Discovery Model with QoS Constraints," in Third International Conference on Semantics, Knowledge and Grid, 2007, pp. 479-482.

[51] W. D. Yu, R. B. Radhakrishna, S. Pingali, and V. Kolluri, "Modeling the Measurements of QoS Requirements in Web Service Systems," Simulation, vol. 83, no. 1, pp. 75-91, 2007.

[52] Y. Kang, "Extended Model Design for Quality Factor Based Web Service Management," in Future Generation Communication and Networking (FGCN), 2007, pp. 484-487.

[53] N. Artaiam and T. Senivongse, "Enhancing Service-Side QoS Monitoring for Web Services," in Ninth ACIS International Conference on Software Engineering, Artificial Intelligence, Networking, and Parallel/Distributed Computing (SNPD), 2008, pp. 765-770.

[54] E. Giallonardo and E. Zimeo, "More semantics in qos matching," in IEEE International Conference on Service-Oriented Computing and Applications (SOCA), 2007, pp. 163-171.

[55] G. Damiano, E. Giallonardo, and E. Zimeo, "onQoS-QL: A Query Language for QoS-Based Service Selection and Ranking," in Service-Oriented Computing-ICSOC 2007 Workshops, 2009, p. 127.

[56] H. Chang and K. Lee, "Quality-Driven Web Service Composition for Ubiquitous Computing Environment," in International Conference on New Trends in Information and Service Science (NISS), 2009, pp. 156-161.

[57] E. Al-Masri and Q. H. Mahmoud, "Understanding web service discovery goals," in IEEE International Conference on Systems, Man and Cybernetics (SMC), 2009, pp. 3714-3719.

[58] E. Al-Masri and Q. H. Mahmoud, "Identifying Client Goals for Web Service Discovery," in IEEE International Conference on Services Computing, 2009, pp. 202-209. 
[59] H. Tong, J. Cao, S. Zhang, and Y. Mou, "A fuzzy evaluation system for web services selection using extended QoS model," Kybernetes, vol. 38, no. 3/4, pp. 513-521, 2009.

[60] Z. Balfagih and M. F. Hassan, "Quality Model for Web Services from Multi-stakeholders' Perspective," in Proceedings of the International Conference on Information Management and Engineering, 2009, pp. 287-291.

[61] S. Li and J. Zhou, "The WSMO-QoS Semantic Web Service Discovery Framework," in International Conference on Computational Intelligence and Software Engineering, 2009, pp. 1-5.

[62] K. K. Reddy, K. Maralla, G. Raj Kumar, and M. Thirumaran, "A greedy approach with criteria factors for QoS based web service discovery," in Proceedings of the 2nd Bangalore Annual Compute Conference - COMPUTE, 2009, pp. 12:1-12:5.

[63] R. Mohanty, V. Ravi, and M. R. Patra, "Web-services classification using intelligent techniques," Expert Syst.Appl., vol. 37, no. 7, pp. 5484-5490, 2010.

[64] H. Yang, X. Chen, and S. Liu, "Research and Implementation on QoS Ontology of Web Service-Oriented Composition," in 2010 2nd International Symposium on Information Engineering and Electronic Commerce, 2010, pp. 1-4.

[65] Y. Baocai, Y. Huirong, F. Pengbin, G. Liheng, and L. Mingli, "A framework and QoS based web services discovery," in IEEE International Conference on Software Engineering and Service Sciences, 2010, pp. 755-758.

[66] Z. Pan and J. Baik, "A QOS enhanced framework and trust model for effective web services selection," J.Web Eng., vol. 9, no. 2, pp. 186-204, 2010.

[67] Z. Shu and S. Meina, "An architecture design of life cycle based SLA management," in The 12th International Conference on Advanced Communication Technology (ICACT), 2010, vol. 2, pp. 1351-1355.

[68] Y. Lee, "Quality Control for Business Collaboration Based on SOA Framework," in International Conference on Convergence Information Technology (ICCIT), 2007, pp. 1963-1968.

[69] Y. Lee and G. Yeom, "A Quality Chain Modeling Methodology for Ternary Web Services Quality View," in 5th ACIS International Conference on Software Engineering Research, Management \& Applications (SERA), 2007, pp. 91-97.

[70] Y. Lee and G. Yeom, "A Research for Web Service Quality Presentation Methodology for SOA Framework," in Sixth International Conference on Advanced Language Processing and Web Information Technology (ALPIT), 2007, pp. 434-439.

[71] Y. Lee and G. Yeom, "A Research for Quality Preservation Methodology for SOA Environment," in Eighth ACIS International Conference on Software Engineering, Artificial Intelligence, Networking, and Parallel/Distributed Computing (SNPD), 2007, vol. 2, pp. 429-434. 
[72] Youngkon Lee, "QoS metrics for service level measurement for SOA environment," in 6th International Conference on Advanced Information Management and Service (IMS), 2010, pp. 509-514.

[73] P. Bocciarelli and A. D'Ambrogio, "A model-driven method for describing and predicting the reliability of composite services," Softw. Syst. Model., vol. 10, no. 2, pp. 265-280, 2011.

[74] A. D'Ambrogio, "A Model-driven WSDL Extension for Describing the QoS ofWeb Services," in International Conference on Web Services (ICWS), 2006, pp. 789-796.

[75] J. Qiu and F. Yu, "Research on Semantic Dynamic Service Combination Module," in International Conference on Internet Technology and Applications, 2011, pp. 1-4.

[76] N. Debnath, P. Martellotto, M. Daniele, D. Riesco, and G. Montejano, "A method to evaluate QoS of web services required by a workflow," in 11th International Conference on ITS Telecommunications, 2011, pp. 640-645.

[77] F. Rosenberg, C. Platzer, and S. Dustdar, "Bootstrapping Performance and Dependability Attributes ofWeb Services," in International Conference on Web Services (ICWS), 2006, pp. 205-212.

[78] O. Moser, F. Rosenberg, and S. Dustdar, "Domain-Specific Service Selection for Composite Services," Softw. Eng. IEEE Trans., vol. 38, no. 4, pp. 828-843, 2012.

[79] R. Phalnikar and P. A. Khutade, "Survey of QoS based web service discovery," in World Congress on Information and Communication Technologies, 2012, pp. 657-661.

[80] W. N. Wan Ab. Rahman and F. Meziane, "The Awareness of QoS Consideration for Web Services," in International Business Information Management Association (IBIMA), 2012, pp. 509-516.

[81] J. Pablo Carvallo, X. Franch, and C. Quer, "Managing Non-Technical Requirements in COTS Components Selection," in Proceedings of the 14th IEEE International Requirements Engineering Conference, 2006, pp. 316-321.

[82] T. Erl, Soa: principles of service design, vol. 1. Prentice Hall Upper Saddle River, 2008. 